WSRC-TR-96-0262

\title{
Distribution of Radionuclides in L-Lake Surface Sediments Phase 3 (U)
}

D. L. Dunn

Westinghouse Savannah River Company

Savannah River Technology Center

Environmental Sciences Section

Aiken, SC 29808

August 1996

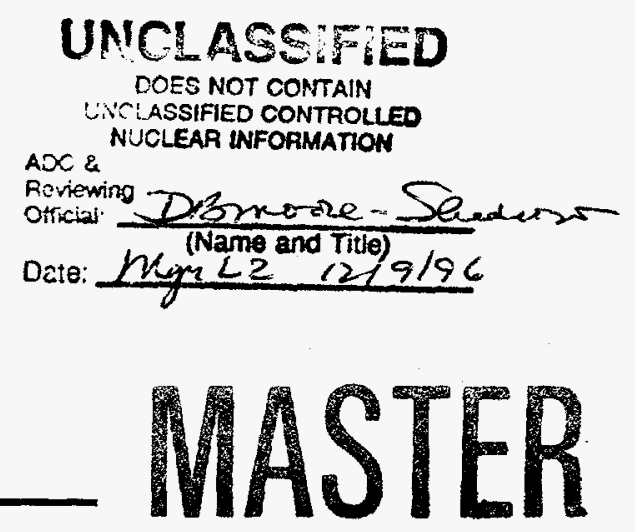

The information contained in this report was developed during the course of work with the U.S. Department of Energy under Contract No. DE-AC09-89SR18035. 


\section{DISCLAIMER}

This report was prepared as an account of work sponsored by an agency of the United States Government. Neither the United States Government nor any agency thereof, nor any of their employees, makes any warranty, express or implied, or assumes any legal liability or responsibility for the accuracy, completeness, or usefulness of any information, apparatus, product, or process disclosed, or represents that its use would not infringe privately owned rights. Reference herein to any specific commercial product, process, or service by trade name, trademark, manufacturer, or otherwise does not necessarily constitute or imply its endorsement, recommendation, or favoring by the United States Government or any agency thereof. The views and opinions of authors expressed herein do not necessarily state or reflect those of the United States Government or any agency thereof.

This report has been reproduced directly from the best available copy.

Available to DOE and DOE contractors from the Office of Scientific and Technical Information, P.O. Box 62, Oak Ridge, TN 37831; prices available from (615) 576-8401.

Available to the public from the National Technical Information Service, U.S. Department of Commerce; 5285 Port Royal Road, Springfield, VA 22161. 


\section{DISCLAMMER}

Portions of this document may be illegible in electronic image products. Images are produced from the best available original document. 


\section{Table of Contents}

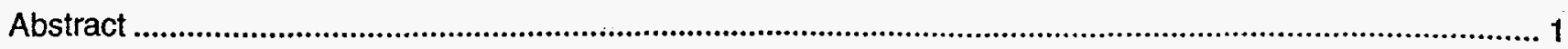

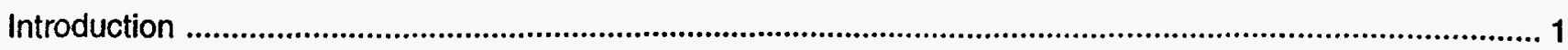

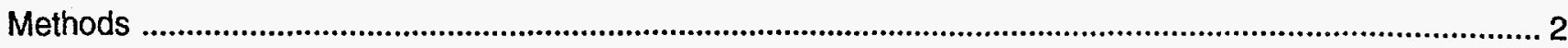

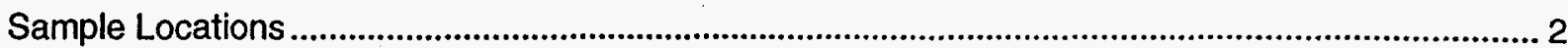

In-Situ Measurements of Radionuclide Distribution in the L-Lake Basin ........................................ 2

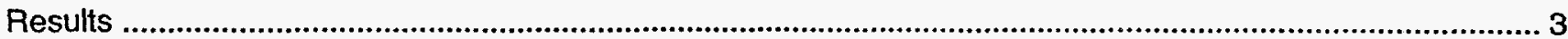

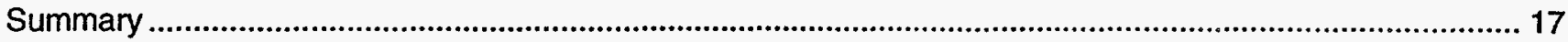

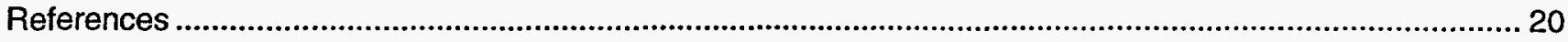

Appendix A Underwater HPGe Data from L-Lake Sediments (1995)

Appendix B Gamma Spectrometry Data - L-Lake Sediments (1995) 


\section{List of Figures}

Figure 1.

Distribution of cesium-137 in L-Lake sediments as determined by an underwater detector. 1996 4

Figure 2.

Current cesium-137 surface model (based on 1995 and 1996 sampling programs) 5

Figure 3.

The 1995 resident scenario cesium-137 15-mrem isodose contours 6

Figure 4.

Cesium-137 isodose contours from 1985 overflight sodium iodide mapping (from Feimster 1992) 7

Figure 5.

Cobalt-60:cesium-137 ratios at 76 locations in L Lake. 1996 8

Figure 6.

Cobalt-60 isodose contours from 1985 EG\&G overflight sodium iodide mapping (from Feimster 1992) 18

\section{List of Tables}

Table 1.

Cesium-137 count rate (counts per minute), depth, and UTM coordinates at each location sampled in L Lake. 1996 9

Table 2.

Results of cobalt-60:cesium-137 ration analysis at 76 waypoints in L Lake.

All results are decay-corrected to April 24, 1996 15 


\title{
Distribution of Radionuclides in L-Lake Surface Sediments Phase 3 (U)
}

\author{
D. L. Dunn \\ Westinghouse Savannah River Company \\ Savannah River Technology Center \\ Environmental Sciences Section \\ Aiken, SC 29808
}

\begin{abstract}
Gamma-emitting radionuclides in L Lake were examined in situ with an underwater High Purity Germanium (HPGe) detector and further studied by retrieving various sediment samples for analysis by HPGe gamma spectrometry. The predominant man-made radionuclide detected was cesium-137. These measurements constituted Phase 3 of a four phase strategy for characterizing L-Lake contaminants. The data provided by these studies will be utilized in the preparation of an Environmental Impact Statement that evaluates the consequences of discontinuing river water pumping to the man-made cooling water reservoirs on the Savannah River Site. A site evaluation report will also be prepared for the L-Lake basin.
\end{abstract}

\section{Introduction}

L Lake is a 400-hectare (1000-acre), man-made reservoir located in the southeastern quadrant of the Savannah River Site (SRS). The lake, constructed in 1985 , was created by impounding the middle reaches of Steel Creek. When completed, it served as a once-through cooling reservoir for $\mathrm{L}$ Reactor. L Reactor was refurbished in the early 1980s and was restarted in 1985 after 13 years of shutdown. The reservoir's purpose was to limit the thermal damage to the lower reaches of Steel Creek caused by secondary cooling water releases.

The operations of $\mathrm{P}$ and L Reactors began in 1954. Secondary cooling water pumped from the Savannah River, through the reactors, and discharged into Steel Creek increased the natural flow rate of 1 cubic meter per second to a maximum of 24 cubic meters per second. This secondary cooling water was released at temperatures as high as 70 degrees centigrade (158 degrees Fahrenheit) to SRS streams. P-Reactor cooling was diverted from Steel Creek to Par Pond in 1963, and L Reactor was placed on standby and shut down in 1968 (Tinney et al. 1986).
As a result of reactor operations from 1954-1968, aquatic and riparian vegetation in Steel Creek were killed, and extensive channel erosion took place. From 1955 to 1973, approximately 284 curies of cesium-137 was released to Steel Creek. Since cesium has a strong affinity for sediments, the majority of the released material was adsorbed to them and deposited with them in the Steel Creek floodplain prior to reaching the Savannah River. An inventory of the cesium-137 in Steel Creek, at 1991 decay-corrected concentrations, estimates 8 curies upstream from $L$ Reactor, 30 curies between L Reactor and the Steel Creek delta, 20 curies in the Steel Creek delta, and 8 curies between the delta and the mouth of Steel Creek at the Savannah River Site boundary, a total of 66 curies in the Steel Creek system (Brisbin et al. 1974; Gladden et al. 1985; Carlton et al. 1992).

During the construction of L Lake, soil removed from the stream bed and floodplain of Steel Creek at the dam site was placed in a planned waste containment pit. The dredged soil was allowed to dry, then leveled and covered with 1.6 meters (m) (5 feet) of clean soil (Zeigler et al. 1985). Approximately 46,400 cubic meters ( 1.6 million cubic feet) 
of contaminated soil was removed from the dam site. Although only the upper $1.2 \mathrm{~m}$ (4 feet) of stream bed material was considered to be contaminated, the stream bed was excavated to a depth of $3 \mathrm{~m}$ (10 feet) (Gladden et al. 1989). This contaminated soil was estimated to contain 0.2 curies of cesium- 137 and 0.02 curies of cobalt- 60 (Zeigler et al. 1985). The buried waste containment pit was covered by the reservoir when the lake was filled (DOE 1984).

In 1985, prior to the filling of L Lake, EG\&G Energy Measurements, Inc. (EG\&G/EM) used aerial gamma survey measurements to establish a baseline radiological survey of the Steel Creek drainage basin. Gamma maps of the area, created from the EG\&G overflight data, confirmed the deposition of man-made radionuclides in the Steel Creek stream bed (Feimster 1992), which was subsequently covered by the reservoir.

In response to the need for a full-scale contaminants screening study, a four-phase sampling program was established. This program was designed to develop a complete and defensible list of contaminants for L Lake and to identify all contaminants of concern (COC) in the sediments of L Lake. During the summer of 1996, the Savannah River Technology Center (SRTC), Environmental Sciences Section (ESS) established and conducted a four-phase program to characterize the current spatial distribution of radioactive and metal contaminants in the sediments of L Lake. Phase 1 of the program sampled surface sediments to identify radioisotopes and metals and determine their concentrations. Phase 2 used vibracore technology to penetrate the surface sediment and provide deeper core sediment samples from the L Lake basin. Phase 3 used a high purity germanium (HPGe) underwater gamma detector in-situ to examine gamma-emitting radionuclides in the surface sediments in order to assess the possibility of sediments redistribution from the stream bed and floodplain at the bottom of the lake. Phase 4 used acoustic impedance technology to map the distribution of floodplain sediments in the basin and to detect buried materials such as vegetation and floodplain sediment covered by a sandy overburden.

The purpose of this study is to begin an evaluation of the potential environmental impacts of reducing or eliminating the flow requirements to the SRS river water distribution system (DOE 1995). There are no plans to restart L Reactor. Without input from the Savannah River, it is predicted that LLake would eventually drain because the watershed above the lake is not sufficiently large enough to maintain it. Allowing L Lake to drain is a costsaving option being considered by DOE. The sediment analysis provides definitive information on the contaminants in L Lake. This information will be considered in the decision on the future of $\mathrm{L}$ Lake. Phase 3 was completed in August 1996 and is discussed in this report. Phase 1, Phase 2, and Phase 4 tasks will be presented in separate reports.

\section{Methods}

\section{Sample Locations}

A Global Positioning System (GPS) roving receiver was used to record the sampling locations. GPS is a satellite-based navigation system developed to provide a consistent; accurate method of simplifying navigation and global positioning. The GPS receiver can determine its present position anywhere on earth with an accuracy to $15 \mathrm{~m}$ (50 feet). L-Lake sampling coordinates are designated as waypoints and are identified throughout this report by Universal Transverse Mercator (UTM) coordinates.

\section{In-Situ Measurements of Radionuclide Distri- bution in the L-Lake Basin}

Phase 3 used an underwater gamma detector for the collection of in-situ data from the lake basin. The measurements of gamma-emitting radioisotopes (primarily cesium-137 and cobalt-60) in surface sediments of $\mathrm{L}$ Lake were conducted from 
May-June 11, 1996. With the HPGe, the count rate is proportional to the radiation levels that were observed during the 1985 overflight when the sediments were not covered by water (Feimster 1992) Therefore, the count-rate profiles generated by the detector are appropriate for comparison with the 1985 gamma mappings.

Grab samples of the bottom sediments were also collected and analyzed with low-level HPGe gamma spectrometry in the Underground Counting Facility. The grab samples were taken to determine the incidence of man-made radionuclides present in the sediments at levels below the detection limit of the underwater HPGe detector. The goal of the HPGe sampling was to determine the edge of the gamma-emitting radionuclide (cesium137 and cobalt-60) contamination in the lake bed and compare it with the contour established in 1985.

The overall equipment has been described in detail in Winn $(1992,1993,1995 a, b)$. The detector was lowered by a winch until its housing rested on the surface sediment. Two- minute counting intervals were made at 192 locations. At each location, the count rate of the $662 \mathrm{keV}$ cesium-137 gamma spectrum was determined and recorded in the field. Geographic position measurements and depths were recorded.

\section{$\underline{\text { Results }}$}

Underwater HPGe detector measurements of the lake-bottom radioactivity were conducted at locations shown in Figure 1. The data from these measurements are presented in Table 1, where each measurement is characterized by its location coordinates, water depth, and cesium-137 count rate.

Various quality checks were used to appraise the measurements. Locations with suspiciously low count rates were recounted after repositioning the detector to ensure that obstructions such as stumps did not prevent it from resting directly on the bot- tom sediments. After each measurement, any sediment sticking to the bottom screen was flushed away to prevent cross-contaminating measurements between sites. Before, during, and after each series of measurements, a cesium-137/cobalt-60 calibration source was used to assure proper detector operation; a total of 52 calibration checks were made during the measurements. All field results were reviewed and refined as appropriate at the laboratory.

The 1996 grid sampling within the old Steel Creek channel and floodplain was used to develop continuous computer-modeled isodose contours. These data were decay-corrected to 1995 and used with the 1995 underwater HPGe data from $L$ Lake. A surface modeling package (TIN or Triangulated Irregular Network) was used to create Figure 2 from these data.

Contour lines, or isolines, are used in Figure 3 to spatially represent the gamma dose from cesium137 contamination as measured by the underwater HPGe detector. These 1995 isodose contours are distributed similarly to the isodose contours measured by the 1985 EG\&G overflights (Figure 4; Feimster 1992).

All count rate data were decay-corrected to 1995 . A dose conversion was performed (see Dum 1995). All dose/point data were corrected to simulate an annual exposure of 15 millirem for a resident living on the lake bed. The resident was assumed to be in residence in a home that provided $20 \%$ shielding 350 days per year, 24 hours per day. The dose interval contours in Figure 3 were generated from known point data. The contour lines were interpolated between known dose/point data.

At the 76 locations in Figure 5, sediment samples were retrieved from the lake bottom. These samples were dried and counted overnight or longer on either the $90 \%$ - or $166 \%$-efficient HPGe detectors in the Underground Counting Facility (Winn et al. 1987). These samples had cesium-137 concentra- 


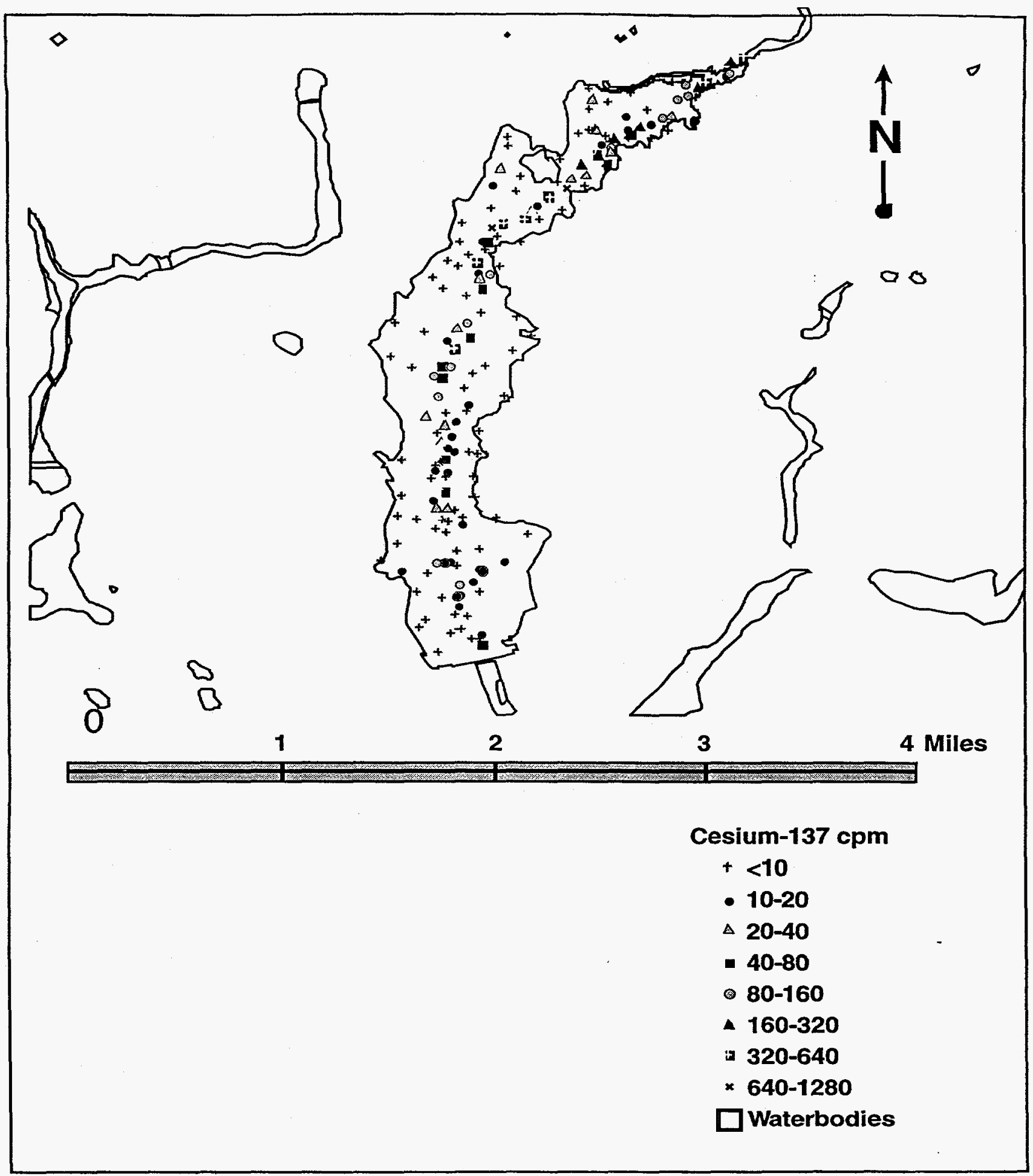

Figure 1. Distribution of cesium-137 in L-Lake sediments as determined by an underwater detector.

tions ranging from 138 to more than 47,000 picoCuries per kilogram ( $\mathrm{pCi} / \mathrm{kg}$ ). Cobalt -60 was the only man-made isotope observed other than cesium-137; it occurred at concentrations two or- ders of magnitude lower than those for cesium137. The cobalt -60 concentrations ranged from 0 to more than $800 \mathrm{pCi} / \mathrm{kg}$ (Table 2). Sample results are from the top few centimeters of sediments. 


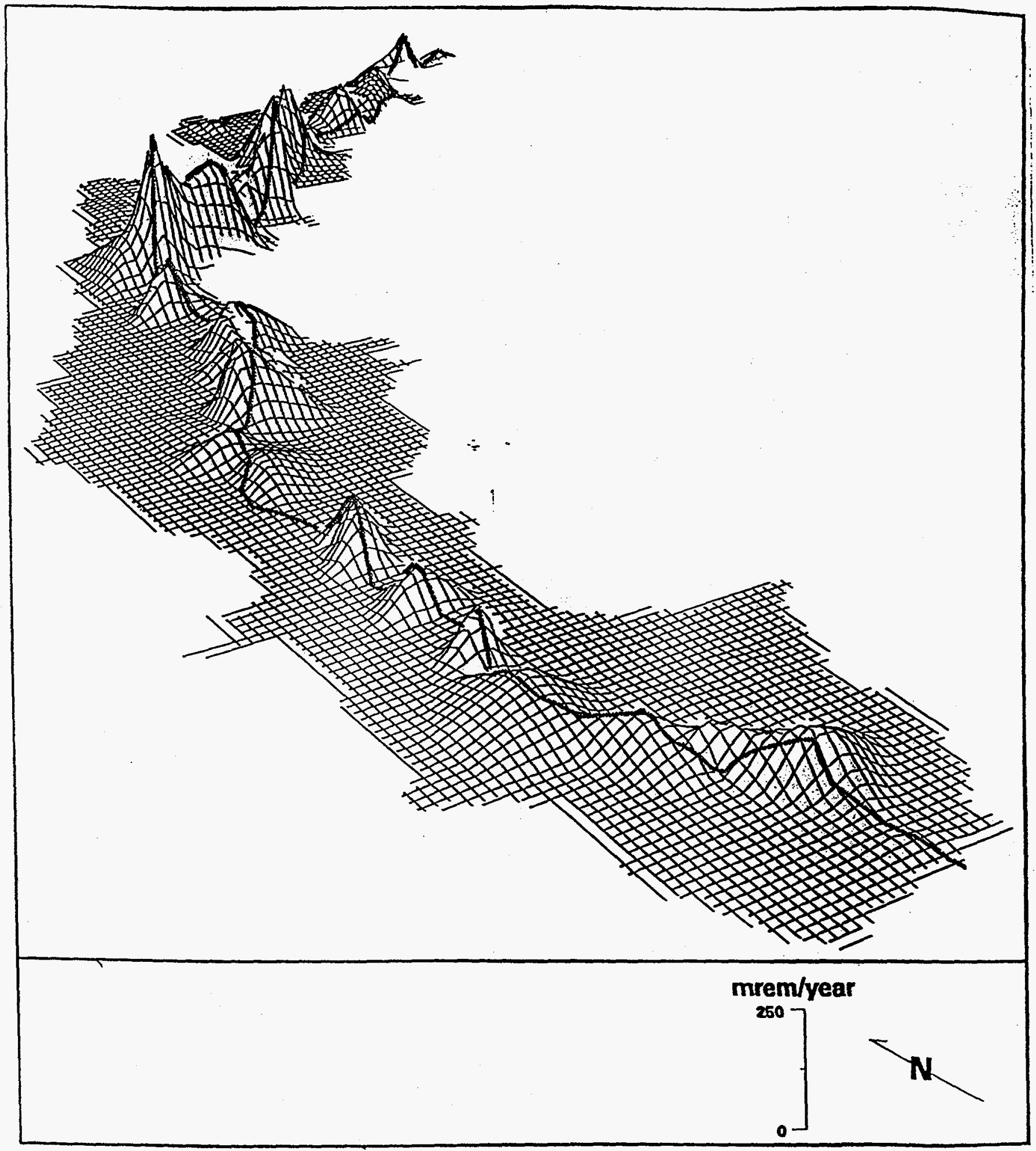

Figure 2. Current cesium-137 surface inodel (based on 1995 and 1996 sampling programs). 

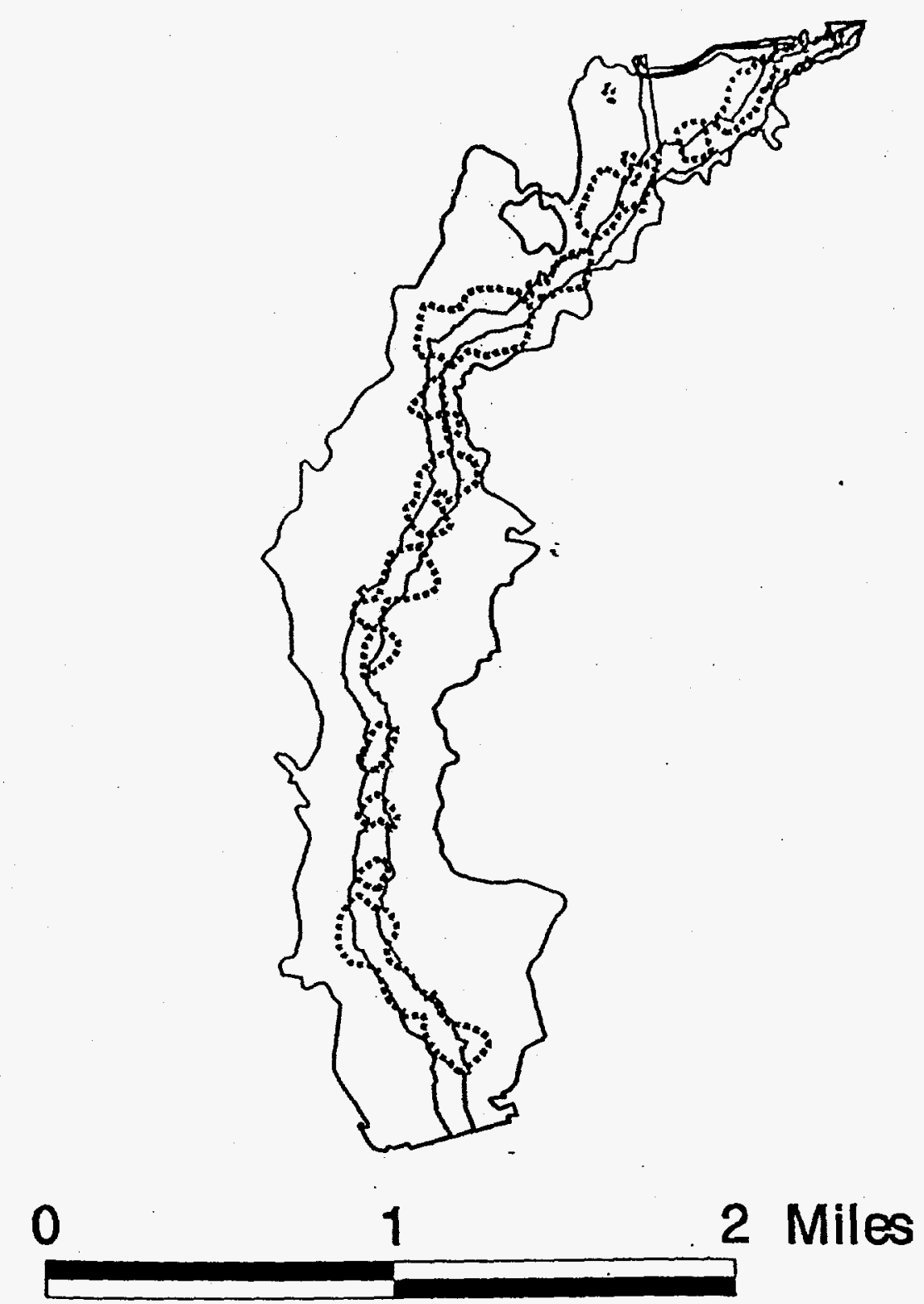

Resident

$\therefore 15$ mrem/year

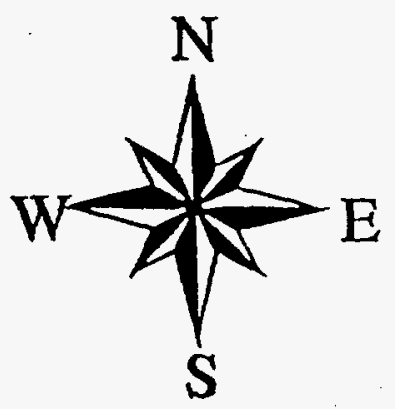

Figure 3. The 1995 resident scenario cesium-137 15-mrem isodose contours. 


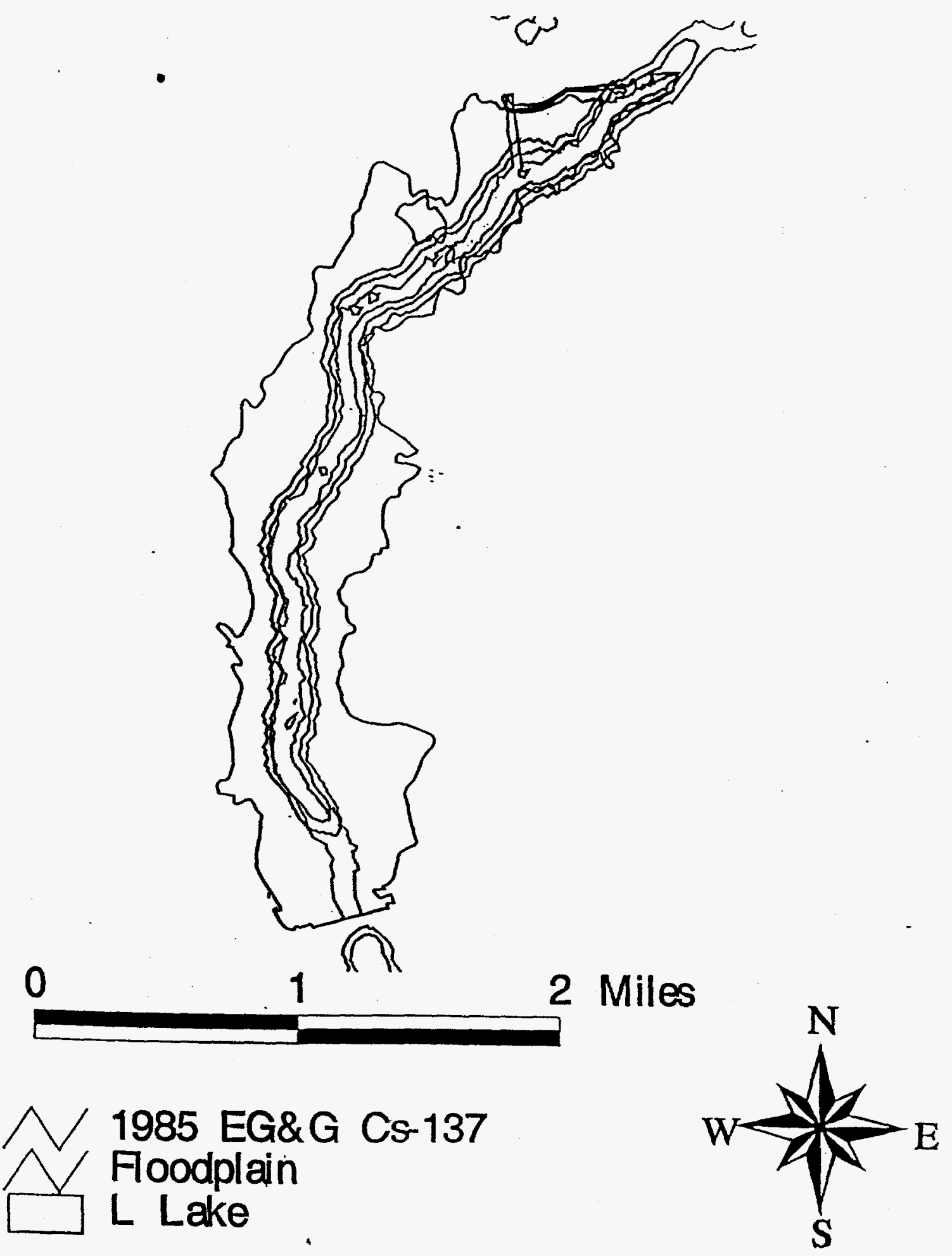

Figure 4. Cesium-137 isodose contours from 1985 overflight sodium iodide mapping (from Feimster 1992). 

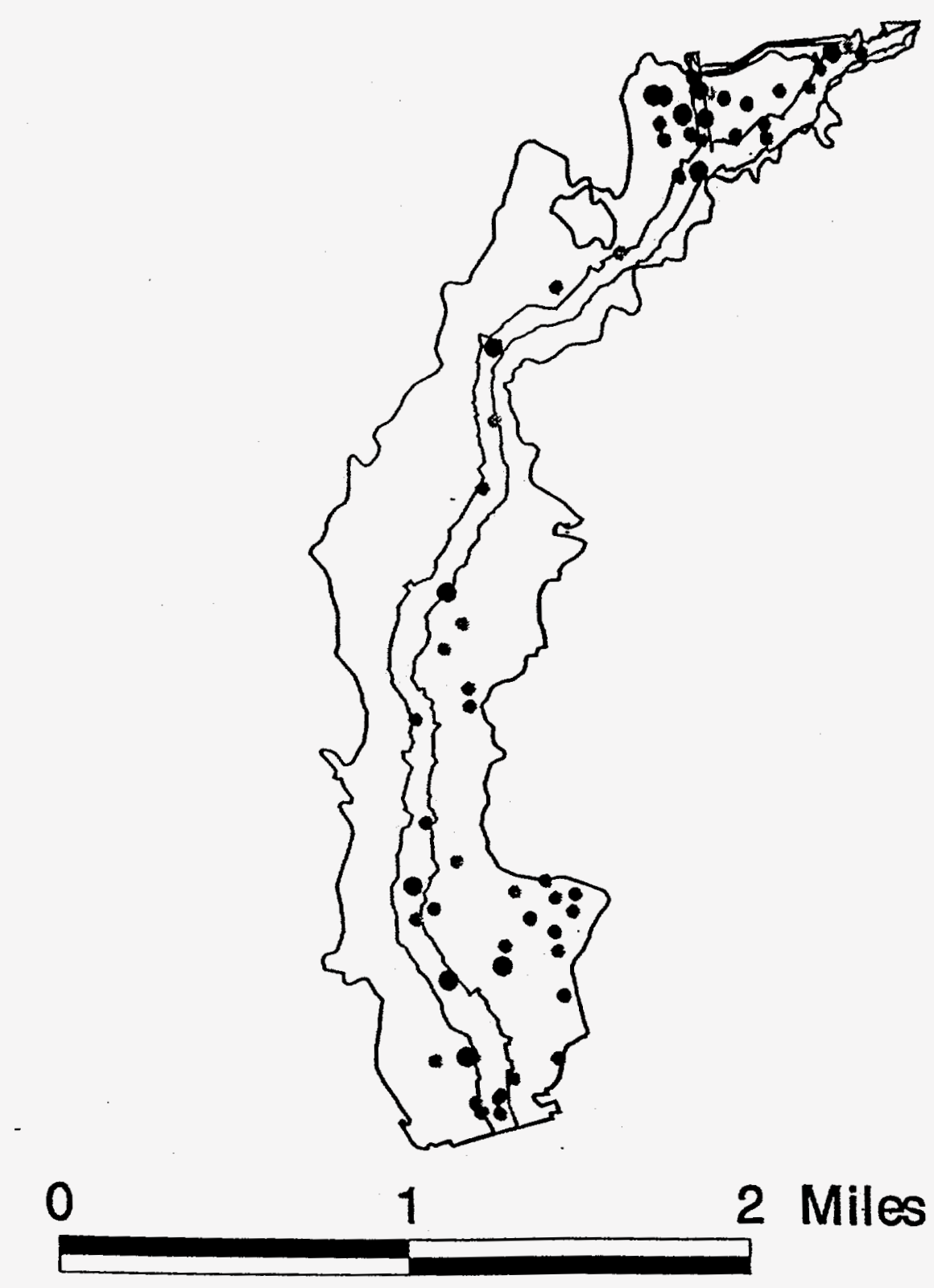

1996 Ca-60/Cs-137 retio

- $0.51-1.05$

- $1.05-1.36$

- $1.36-1.72$

- $1.72-1.87$

- $1.97-6.67$

$\triangle$ Floodplaín

L LAk

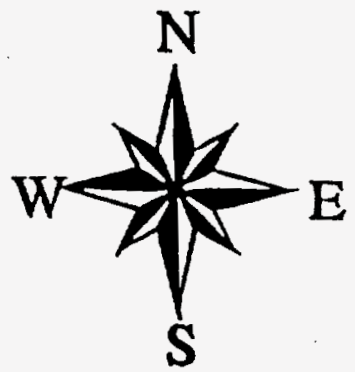

Figure 5. Cobalt-60:cesium-137 ratios at 76 locations in L Lake. 1996. 
Table 1. Cesium-137 count rate (counts per minute), depth, and UTM coordinates at each location sampled in L Lake. 1996. ${ }^{a}$

\begin{tabular}{|c|c|c|c|c|c|c|}
\hline Waypoint & Date & $\begin{array}{l}\text { UTM } \\
\text { north }\end{array}$ & $\begin{array}{l}\text { UTM } \\
\text { east }\end{array}$ & $\begin{array}{l}\text { Depth } \\
\text { (feet) }\end{array}$ & $\begin{array}{l}\text { Cesium-137 } \\
(\mathrm{cpm})^{\mathrm{C}}\end{array}$ & $\begin{array}{l}\text { Error } \\
\text { (cpm) }\end{array}$ \\
\hline \multicolumn{7}{|c|}{ - } \\
\hline 2 & $5 / 14 / 96$ & 3673505 & 441991 & 22 & 254 & $+1-10$ \\
\hline 3 & $5 / 14 / 96$ & 3673555 & 441960 & 22 & 7 & $+1-2$ \\
\hline 4 & $5 / 14 / 96$ & 3673618 & 441868 & $\overline{14}$ & 8 & $+/-2$ \\
\hline 5 & $5 / 14 / 96$ & 3673609 & 441896 & 14 & 24 & $+1-4$ \\
\hline 6 & $5 / 14 / 96$ & 3673781 & 441820 & 9 & 0 & $+1-1$ \\
\hline 7 & $5 / 14 / 96$ & 3673866 & 441858 & 8 & 21 & $+1-3$ \\
\hline 8 & $5 / 14 / 96$ & 3673845 & 441972 & 4 & 4 & $+1-2$ \\
\hline 9 & $5 / 14 / 96$ & 3674208 & 443014 & 8 & 61 & $+/-5$ \\
\hline 10 & $5 / 14 / 96$ & 3674245 & 443015 & 10 & 364 & $+/-15$ \\
\hline 11 & $5 / 14 / 96$ & 3674200 & 442913 & 12 & 197 & $+/-12$ \\
\hline 12 & $5 / 14 / 96$ & 3674105 & 442914 & 4 & 84 & $+1-7$ \\
\hline 13 & $5 / 14 / 96$ & 3674065 & 442882 & 12 & 20 & $+/-4$ \\
\hline 14 & $5 / 14 / 96$ & 3674114 & 442765 & 3 & 0 & $+/-1$ \\
\hline 15 & $5 / 14 / 96$ & 3674023 & 442720 & 14 & 332 & $+/-16$ \\
\hline 16 & $5 / 14 / 96$ & 3673970 & 442662 & 14 & 234 & $+/-12$ \\
\hline 17 & $5 / 14 / 96$ & 3673998 & 442561 & 6 & 103 & $+/-8$ \\
\hline 18 & $5 / 14 / 96$ & 3673902 & 442573 & 12 & 136 & $+1-9$ \\
\hline 19 & $5 / 14 / 96$ & 3673727 & 442468 & 11 & $\overline{37}$ & $+1-4$ \\
\hline 20 & $5 / 14 / 96$ & 3673780 & 442269 & 6 & 4 & $+/-3$ \\
\hline 21 & $5 / 14 / 96$ & 3673656 & 442306 & 16 & 13 & $+/-3$ \\
\hline 22 & $5 / 14 / 96$ & 3673539 & 442124 & 17 & 2 & $+/-3$ \\
\hline 23 & $5 / 14 / 96$ & 3673598 & 442132 & 16 & 13 & $+/-3$ \\
\hline 24 & $5 / 14 / 96$ & 3673529 & 442012 & 21 & 268 & $+/-10$ \\
\hline 25 & $5 / 14 / 96$ & 3673398 & 441891 & 24 & 550 & $+1-20$ \\
\hline 26 & $5 / 14 / 96$ & 3673380 & 441891 & 22 & 163 & $+/-13$ \\
\hline 27 & $5 / 14 / 96$ & 3673320 & 441978 & 19 & 45 & $+/-5$ \\
\hline 28 & $5 / 14 / 96$ & 3673302 & 441961 & 14 & 7 & $+1-3$ \\
\hline 29 & $5 / 14 / 96$ & 3673412 & 442005 & 17 & 31 & $+1-4$ \\
\hline 30 & $5 / 14 / 96$ & 3673466 & 441980 & 21 & 134 & $+/-7$ \\
\hline 31 & $5 / 14 / 96$ & 3673483 & 441929 & 18 & 15 & $+/-4$ \\
\hline 32 & $5 / 14 / 96$ & 3673600 & 441836 & 13 & 6 & $+/-3$ \\
\hline 33 & $5 / 14 / 96$ & 3673718 & 442111 & 8 & 15 & $+1-4$ \\
\hline$\overline{34}$ & $5 / 14 / 96$ & 3673638 & 442222 & 18 & 298 & $+/-15$ \\
\hline 35 & $5 / 14 / 96$ & 3673717 & 442390 & 16 & 110 & $+/-9$ \\
\hline
\end{tabular}

a See Appendix A for 1995 underwater HPGe data.

b Universal Transverse Mercator.

c Counts per minute. 
Table 1 (continued). Cesium-137 count rate (counts per minute), depth, and UTM coordinates at each location sampled in L Lake. 1996."

\begin{tabular}{|c|c|c|c|c|c|c|}
\hline Waypoint & Date & $\begin{array}{l}\text { UTMb } \\
\text { north }\end{array}$ & $\begin{array}{l}\text { UTM } \\
\text { east }\end{array}$ & $\begin{array}{l}\text { Depth } \\
\text { (feet) }\end{array}$ & $\begin{array}{l}\text { Cesium-137 } \\
\text { (cpm)c }\end{array}$ & $\begin{array}{l}\text { Error } \\
\text { (cpm) }\end{array}$ \\
\hline \multicolumn{7}{|c|}{ 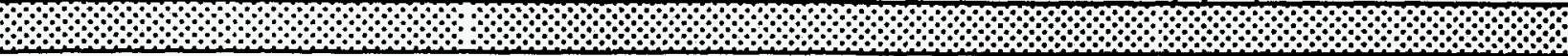 } \\
\hline 36 & $5 / 14 / 96$ & 3673880 & 442505 & 16 & 146 & $+1-10$ \\
\hline 37 & $5 / 20 / 96$ & 3673567 & 442149 & $\overline{17}$ & 47 & $+1-4$ \\
\hline 39 & $5 / 20 / 96$ & 3670021 & 440802 & 21 & 0 & $+/-2$ \\
\hline 40 & $5 / 20 / 96$ & 3670200 & 440646 & 41 & 10 & $+/-3$ \\
\hline 41 & $5 / 20 / 96$ & 3670322 & 440665 & 59 & 286 & $+1-14$ \\
\hline 42 & $5 / 20 / 96$ & 3670361 & 440790 & 53 & 7 & $+/-3$ \\
\hline 43 & $5 / 20 / 96$ & 3670296 & 440850 & 25 & 8 & $+1-2$ \\
\hline 44 & $5 / 20 / 96$ & 3670386 & 440737 & $\overline{53}$ & 21 & $+/-4$ \\
\hline 45 & $5 / 20 / 96$ & 3670283 & 440693 & 55 & 8 & $+1-3$ \\
\hline 46 & $5 / 20 / 96$ & 3670268 & -440741 & 55 & 10 & $+1-3$ \\
\hline 47 & $5 / 20 / 96$ & 3670388 & 440656 & 57 & 38 & $+/-5$ \\
\hline 48 & $5 / 21 / 96$ & 3671277 & 440900 & 38 & 12 & $+1-2$ \\
\hline 49 & $5 / 21 / 96$ & 3671189 & 440884 & $\overline{31}$ & 6 & $+/-3$ \\
\hline 50 & $5 / 21 / 96$ & 3671113 & 440812 & 38 & 15 & $+1-3$ \\
\hline 51 & $5 / 21 / 96$ & 3670992 & 440783 & 47 & 14 & $+/-4$ \\
\hline 52 & $5 / 21 / 96$ & 3670903 & 440750 & 49 & $\overline{11}$ & $+/-7$ \\
\hline 53 & $5 / 21 / 96$ & 3670820 & 440706 & 55 & 65 & $+/-5$ \\
\hline 54 & $5 / 21 / 96$ & 3670756 & 440666 & 48 & 3 & $+1-2$ \\
\hline 55 & $5 / 21 / 96$ & 3669265 & 440981 & $\overline{71}$ & 1 & $+/-2$ \\
\hline 56 & $5 / 21 / 96$ & 3669314 & 440993 & 66 & $\overline{18}$ & $+/-5$ \\
\hline 57 & $5 / 21 / 96$ & 3670442 & 440629 & 57 & 19 & $+/-5$ \\
\hline 58 & $5 / 21 / 96$ & 3670582 & 440665 & 57 & 238 & $+/-11$ \\
\hline 59 & $5 / 21 / 96$ & 3670681 & 440747 & 51 & 12 & $+1-3$ \\
\hline 60 & $5 / 21 / 96$ & 3670787 & 440690 & 50 & 10 & $+/-3$ \\
\hline 62 & $5 / 21 / 96$ & 3669543 & 440830 & 64 & 11 & $+/-3$ \\
\hline 63 & $5 / 21 / 96$ & 3669488 & 440980 & 68 & 256 & $+/-14$ \\
\hline 64 & $5 / 21 / 96$ & 3669669 & 440983 & 62 & 0 & $+1-2$ \\
\hline 65 & $5 / 21 / 96$ & 3669850 & 440989 & 57 & 12 & $+/-5$ \\
\hline 66 & $5 / 21 / 96$ & 3669933 & 440763 & 61 & 13 & $+/-4$ \\
\hline 67 & $5 / 21 / 96$ & 3670175 & 440728 & 39 & 5 & $+/-3$ \\
\hline 68 & $5 / 21 / 96$ & 3670235 & 440853 & 44 & 16 & $+/-4$ \\
\hline 69 & $5 / 21 / 96$ & 3670519 & 440726 & 55 & 47 & $+1-6$ \\
\hline
\end{tabular}

a See Appendix A for 1995 underwater HPGe data.

b Universal Transverse Mercator.

c Counts per minute. 
Table 1 (continued). Cesium-137 count rate (counts per minute), depth, and UTM coordinates at each location sampled in L Lake. $1996 .{ }^{a}$

\begin{tabular}{|c|c|c|c|c|c|c|}
\hline Waypoint & Date & $\begin{array}{l}\text { UTMb } \\
\text { north }\end{array}$ & $\begin{array}{l}\text { UTM } \\
\text { east }\end{array}$ & $\begin{array}{l}\text { Depth } \\
\text { (feet) }\end{array}$ & $\begin{array}{l}\text { Cesium-137 } \\
\text { (cpm) }\end{array}$ & $\begin{array}{l}\text { Error } \\
\text { (cpm) }\end{array}$ \\
\hline & & & & & & \\
\hline 70 & $5 / 21 / 96$ & 3670658 & 440722 & 50 & 7 & $+1-2$ \\
\hline 71 & $5 / 21 / 96$ & 3669849 & 440969 & 58 & 20 & $+/-4$ \\
\hline 72 & $5 / 21 / 96$ & 3669738 & 440935 & 62 & 13 & $+1-3$ \\
\hline 73 & $5 / 23 / 96$ & 3669600 & 440889 & 65 & 180 & $+/-9$ \\
\hline 74 & $5 / 23 / 96$ & 3673295 & 441779 & 24 & 194 & $+/-16$ \\
\hline 75 & $5 / 23 / 96$ & 3673229 & 441806 & 23 & 28 & $+1-4$ \\
\hline 76 & $5 / 23 / 96$ & 3673170 & 441721 & 26 & 37 & $+/-6$ \\
\hline 77 & $5 / 23 / 96$ & 3673093 & 441670 & 25 & 706 & $+/-24$ \\
\hline 78 & $5 / 23 / 96$ & 3673029 & 441524 & 30 & 329 & $+/-11$ \\
\hline 79 & $5 / 23 / 96$ & 3673049 & 441513 & 25 & 59 & $+1-6$ \\
\hline 80 & $5 / 23 / 96$ & 3672952 & 441424 & 23 & 15 & $+/-4$ \\
\hline 82 & $5 / 23 / 96$ & 3672911 & 441404 & 32 & 288 & $+/-18$ \\
\hline 83 & $5 / 23 / 96$ & 3672847 & 441330 & 33 & 510 & $+/-20$ \\
\hline 84 & $5 / 23 / 96$ & 3672815 & 441179 & 34 & 431 & $+/-14$ \\
\hline 85 & $5 / 23 / 96$ & 3672691 & 441127 & 28 & 9 & $+/-3$ \\
\hline 86 & $5 / 23 / 96$ & 3672764 & 441076 & 36 & 825 & $+1-27$ \\
\hline 87 & $5 / 23 / 96$ & 3673546 & 441201 & 10 & 9 & $+/-3$ \\
\hline 88 & $5 / 23 / 96$ & 3673463 & 441211 & 8 & 4 & $+1-2$ \\
\hline 89 & $5 / 23 / 96$ & 3673273 & 441163 & 15 & 33 & $+/-9$ \\
\hline 90 & $5 / 23 / 96$ & 3673129 & 441091 & 23 & 17 & $+/-4$ \\
\hline 91 & $5 / 23 / 96$ & 3672937 & 441088 & 25 & 9 & $+/-3$ \\
\hline 92 & $5 / 23 / 96$ & 3672794 & 441008 & 33 & 275 & $+/-15$ \\
\hline 93 & $5 / 23 / 96$ & 3672647 & 441052 & 39 & 56 & $+/-6$ \\
\hline 94 & $5 / 23 / 96$ & 3672653 & 441011 & 30 & 11 & $+/-2$ \\
\hline 95 & $5 / 23 / 96$ & 3672478 & 440969 & 40 & 366 & $+/-16$ \\
\hline 96 & $5 / 23 / 96$ & 3672245 & 441010 & 42 & 66 & $+1-6$ \\
\hline 97 & $5 / 23 / 96$ & 3672101 & 440967 & $\overline{44}$ & 292 & $+/-15$ \\
\hline 98 & $5 / 23 / 96$ & 3671959 & 440879 & 45 & 154 & $+/-10$ \\
\hline 99 & $5 / 23 / 96$ & 3671833 & 440917 & 45 & 43 & $+/-6$ \\
\hline 100 & $5 / 23 / 96$ & 3671738 & 440784 & 47 & 440 & $+/-18$ \\
\hline 101 & $5 / 23 / 96$ & 3671592 & 440754 & 48 & 84 & $+/-7$ \\
\hline 102 & $5 / 23 / 96$ & 3671500 & 440696 & 49 & 73 & $+/-14$ \\
\hline 103 & $5 / 23 / 96$ & 3671343 & 440656 & 51 & 150 & $+1-9$ \\
\hline 104 & $5 / 23 / 96$ & 3671159 & 440588 & 54 & 25 & $+/-3$ \\
\hline
\end{tabular}

a See Appendix A for 1995 underwater HPGe data.

b Universal Transverse Mercator.

c Counts per minute. 
Table 1 (continued). Cesium-137 count rate (counts per minute), depth, and UTM coordinates at each location sampled in L Lake. 1996. ${ }^{\text {a }}$

\begin{tabular}{|c|c|c|c|c|c|c|}
\hline Waypoint & Date & $\begin{array}{l}\text { UTMb } \\
\text { north }\end{array}$ & $\begin{array}{l}\text { UTM } \\
\text { east }\end{array}$ & $\begin{array}{l}\text { Depth } \\
\text { (feet) }\end{array}$ & $\begin{array}{l}\text { Cesium-137 } \\
(\mathrm{cpm})^{c}\end{array}$ & $\begin{array}{l}\text { Error } \\
\text { (cpm) }\end{array}$ \\
\hline an & 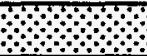 & & 6 & $\beta$ & 6 & n \\
\hline 105 & $6 / 5 / 96$ & 3669263 & 440915 & 53 & 3 & $+1-2$ \\
\hline 106 & $6 / 5 / 96$ & 3669350 & 440840 & 34 & $\overline{0}$ & $+/-2$ \\
\hline 107 & $6 / 5 / 96$ & 3669452 & 440879 & 45 & 4 & $+/-2$ \\
\hline 108 & $6 / 5 / 96$ & 3669639 & 440832 & 67 & 16 & $+1-4$ \\
\hline 109 & $6 / 5 / 96$ & 3669668 & 440840 & 69 & 211 & $+/-16$ \\
\hline 110 & $6 / 5 / 96$ & 3669729 & 440828 & 67 & 95 & $+/-6$ \\
\hline 111 & $6 / 5 / 96$ & 3669793 & 440762 & 68 & 167 & $+/-10$ \\
\hline 112 & $6 / 5 / 96$ & 3669881 & 440812 & 60 & 7 & $+1-3$ \\
\hline 113 & $6 / 5 / 96$ & 3669921 & 440640 & 65 & 105 & $+1-8$ \\
\hline 114 & $6 / 5 / 96$ & 3669924 & 440728 & 67 & 13 & $+1-3$ \\
\hline 115 & $6 / 5 / 96$ & 3670074 & 440631 & 66 & 172 & $+/-11$ \\
\hline 116 & $6 / 5 / 96$ & 3670710 & 440646 & 55 & 11 & $+/-4$ \\
\hline 117 & $6 / 5 / 96$ & 3670839 & 440664 & 60 & 272 & $+/-12$ \\
\hline 118 & $6 / 5 / 96$ & 3670860 & 440784 & 49 & 15 & $+1-4$ \\
\hline 119 & $6 / 5 / 96$ & 3670939 & 440694 & 56 & 279 & $+/-13$ \\
\hline 120 & $6 / 5 / 96$ & 3671020 & 440667 & 58 & 8 & $+/-2$ \\
\hline 121 & $6 / 5 / 96$ & 3671088 & 440726 & 55 & 28 & $+1-5$ \\
\hline 122 & $6 / 5 / 96$ & 3671203 & 440725 & 51 & 5 & $+/-3$ \\
\hline 123 & $6 / 5 / 96$ & 3671218 & 440890 & 42 & 7 & $+1-3$ \\
\hline 124 & $6 / 5 / 96$ & 3669215 & 440999 & 74 & 45 & $+/-4$ \\
\hline 125 & $6 / 5 / 96$ & 3671401 & 440866 & 33 & 10 & $+/-2$ \\
\hline 126 & $6 / 5 / 96$ & 3671523 & 440637 & 52 & 144 & $+/-9$ \\
\hline 127 & $6 / 5 / 96$ & 3671594 & 440700 & 54 & 70 & $+/-7$ \\
\hline 128 & $6 / 5 / 96$ & 3671805 & 440746 & 45 & 11 & $+1-3$ \\
\hline 129 & $6 / 5 / 96$ & 3671921 & 440827 & 40 & 23 & $+/-4$ \\
\hline 130 & $6 / 5 / 96$ & 3671990 & 440941 & 46 & 206 & $+/-11$ \\
\hline 131 & $6 / 5 / 96$ & 3672045 & 440993 & 37 & 4 & $+1-2$ \\
\hline 132 & $6 / 5 / 96$ & 3672139 & 441023 & 44 & 257 & $+/-12$ \\
\hline 133 & $6 / 5 / 96$ & 3672374 & 441059 & 42 & 86 & $+1-7$ \\
\hline 134 & $6 / 5 / 96$ & 3672539 & 440904 & 32 & 8 & $+1-3$ \\
\hline 135 & $6 / 5 / 96$ & 3672339 & 440993 & 42 & 31 & $+/-3$ \\
\hline 136 & $6 / 5 / 96$ & 3672369 & 440984 & 40 & 15 & $+/-3$ \\
\hline 137 & $6 / 5 / 96$ & 3672577 & 441030 & 39 & 2 & $+/-2$ \\
\hline 138 & $6 / 11 / 96$ & 3674016 & 442470 & 3 & 3 & $+1-2$ \\
\hline
\end{tabular}

a See Appendix A for 1995 underwater HPGe data.

b Universal Transverse Mercator.

c Counts per minute. 
Table 1 (continued). Cesium-137 count rate (counts per minute), depth, and UTM coordinates at each location sampled in L Lake. 1996.

\begin{tabular}{|c|c|c|c|c|c|c|}
\hline Waypoint & Date & $\begin{array}{l}\text { UTMb } \\
\text { north }\end{array}$ & $\begin{array}{l}\text { UTM } \\
\text { east }\end{array}$ & $\begin{array}{l}\text { Depth } \\
\text { (feet) }\end{array}$ & $\begin{array}{l}\text { Cesium-137 } \\
(\mathrm{cpm})^{\mathrm{c}}\end{array}$ & $\begin{array}{l}\text { Error } \\
\text { (cpm) }\end{array}$ \\
\hline$\cdots$ & & 6 & & 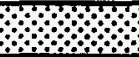 & blo & m \\
\hline 139 & $6 / 11 / 96$ & 3673925 & 442166 & 4 & 4 & $+1-2$ \\
\hline 140 & $6 / 11 / 96$ & 3673958 & 441818 & $\overline{3}$ & 3 & $+/-2$ \\
\hline 141 & $6 / 11 / 96$ & 3673567 & 441751 & 11 & 4 & $+1-2$ \\
\hline 142 & $6 / 11 / 96$ & 3673354 & 441607 & 7 & 9 & $+/-3$ \\
\hline 143 & $6 / 11 / 96$ & 3673152 & 441587 & 10 & 6 & $+/-3$ \\
\hline 144 & $6 / 11 / 96$ & 3673202 & 441304 & $\overline{3}$ & 3 & $+/-2$ \\
\hline 145 & $6 / 11 / 96$ & 3673080 & 441262 & 9 & 3 & $+/-2$ \\
\hline 146 & $6 / 11 / 96$ & 3672808 & 440853 & 9 & 2 & $+/-2$ \\
\hline 147 & $6 / 11 / 96$ & 3672654 & 440841 & 8 & 4 & $+/-2$ \\
\hline 148 & $6 / 11 / 96$ & 3672490 & 440747 & 6 & 4 & $+/-3$ \\
\hline 149 & $6 / 11 / 96$ & 3672341 & 440630 & 10 & 1 & $+/-2$ \\
\hline 150 & $6 / 11 / 96$ & 3671964 & 440346 & 7 & 6 & $+/-2$ \\
\hline 151 & $6 / 11 / 96$ & 3671682 & 440318 & 6 & 1 & $+/-2$ \\
\hline 152 & $6 / 11 / 96$ & 3671575 & 440476 & 35 & $\overline{3}$ & $+/-2$ \\
\hline 153 & $6 / 11 / 96$ & 3671883 & 440571 & 42 & 9 & $+/-3$ \\
\hline 154 & $6 / 11 / 96$ & 3672243 & 440717 & 14 & 10 & $+1-3$ \\
\hline 155 & $6 / 11 / 96$ & 3672436 & 440823 & 10 & 2 & $+/-2$ \\
\hline 156 & $6 / 11 / 96$ & 3672188 & 440883 & 23 & 9 & $+/-3$ \\
\hline 157 & $6 / 11 / 96$ & 3671342 & 441175 & $\overline{3}$ & 2 & $+1-2$ \\
\hline 158 & $6 / 11 / 96$ & 3671598 & 441031 & 13 & 2 & $+1-2$ \\
\hline 159 & $6 / 11 / 96$ & 3671722 & 441241 & 7 & 4 & $+/-2$ \\
\hline 160 & $6 / 11 / 96$ & 3671843 & 441382 & 6 & 1 & $+/-2$ \\
\hline 161 & $6 / 11 / 96$ & 3672007 & 441263 & 6 & 2 & $+1-2$ \\
\hline 162 & $6 / 11 / 96$ & 3672434 & 441143 & $\overline{14}$ & 3 & $+1-2$ \\
\hline 163 & $6 / 11 / 96$ & 3672642 & 441306 & 3 & 4 & $+/-3$ \\
\hline 164 & $6 / 11 / 96$ & 3672832 & 441450 & 8 & 3 & $+/-3$ \\
\hline 165 & $6 / 11 / 96$ & 3672927 & 441617 & 5 & 0 & $+1-2$ \\
\hline 166 & $6 / 11 / 96$ & 3673103 & 441807 & 16 & 1 & $+/-2$ \\
\hline 167 & $6 / 11 / 96$ & 3673538 & 442311 & 4 & 4 & $+1-3$ \\
\hline 168 & $6 / 11 / 96$ & 3673609 & 442427 & 11 & 6 & $+/-3$ \\
\hline 169 & $6 / 11 / 96$ & 3673699 & 442642 & 7 & 19 & $+/-4$ \\
\hline 170 & $6 / 11 / 96$ & 3673892 & 442658 & $\overline{6}$ & 7 & $+/-2$ \\
\hline 171 & $6 / 11 / 96$ & 3671031 & 440976 & 23 & 5 & $+/-3$ \\
\hline
\end{tabular}

a See Appendix A for 1995 underwater HPGe data.

b Universal Transverse Mercator.

c Counts per minute. 
Table 1 (continued). Cesium-137 count rate (counts per minute), depth, and UTM coordinates at each location sampled in L Lake. 1996.

\begin{tabular}{|c|c|c|c|c|c|c|}
\hline Waypoint & Date & $\begin{array}{l}\text { UTM }^{b} \\
\text { north }\end{array}$ & $\begin{array}{l}\text { UTM } \\
\text { east }\end{array}$ & $\begin{array}{l}\text { Depth } \\
\text { (feet) }\end{array}$ & $\begin{array}{l}\text { Cesium-137 } \\
\text { (cpm) }\end{array}$ & $\begin{array}{l}\text { Error } \\
(\mathrm{cpm})\end{array}$ \\
\hline की & & nl & $\beta$ & n & hlol & n \\
\hline 173 & $6 / 11 / 96$ & 3670865 & 440903 & 16 & 1 & $+1-2$ \\
\hline 174 & $6 / 11 / 96$ & 3670649 & 440939 & $\overline{13}$ & 3 & $+1-2$ \\
\hline 175 & $6 / 11 / 96$ & 3670473 & 440949 & 13 & 7 & $+1-3$ \\
\hline 176 & $6 / 11 / 96$ & 3670289 & 441116 & 12 & $\overline{1}$ & $+1-2$ \\
\hline 177 & $6 / 11 / 96$ & 3670157 & 441348 & 12 & 7 & $+1-2$ \\
\hline 178 & $6 / 11 / 96$ & 3669974 & 441254 & 2 & -1 & $+1-2$ \\
\hline 179 & $6 / 11 / 96$ & 3669940 & 441179 & 21 & 18 & $+1-4$ \\
\hline 180 & $6 / 11 / 96$ & 3670039 & 440979 & 31 & 4 & $+1-2$ \\
\hline 181 & $6 / 11 / 96$ & 3670791 & 440410 & 14 & 4 & $+1-2$ \\
\hline 182 & $6 / 11 / 96$ & 3670501 & 440390 & 22 & 8 & $+1-3$ \\
\hline 183 & $6 / 11 / 96$ & 3670325 & 440365 & $\overline{14}$ & 5 & $+1-2$ \\
\hline 184 & $6 / 11 / 96$ & 3670286 & 440504 & 55 & 7 & $+1-3$ \\
\hline 185 & $6 / 11 / 96$ & 3670080 & 440359 & 13 & $\overline{3}$ & $+/-2$ \\
\hline 186 & $6 / 11 / 96$ & 3669940 & 440233 & 10 & 1 & $+1-2$ \\
\hline 187 & $6 / 11 / 96$ & 3669861 & 440397 & $\overline{19}$ & 16 & $+/-4$ \\
\hline 188 & $6 / 11 / 96$ & 3669825 & 440583 & 31 & 5 & $+1-3$ \\
\hline 189 & $6 / 11 / 96$ & 3669670 & 440509 & 10 & 9 & $+/-3$ \\
\hline 190 & $6 / 11 / 96$ & 3669625 & 440688 & 27 & 5 & $+1-3$ \\
\hline 191 & $6 / 11 / 96$ & 3669476 & 440797 & 28 & 4 & $+1-2$ \\
\hline 192 & $6 / 11 / 96$ & 3669428 & 440570 & 13 & 3 & $+/-3$ \\
\hline 193 & $6 / 11 / 96$ & 3669360 & 440523 & 9 & 4 & $+/-3$ \\
\hline 194 & $6 / 11 / 96$ & 3669159 & 440663 & 23 & 2 & $+1-2$ \\
\hline 195 & $6 / 11 / 96$ & 3669314 & 440756 & 32 & 2 & $+1-3$ \\
\hline
\end{tabular}

a See Appendix A for 1995 underwater HPGe data.

b Universal Transverse Mercator.

c Counts per minute.

Overall, the data show results similar to those from the 1995 survey (Dunn et al. 1995), with cobalt60:cesium-137 ratios clustering near $1-2 \%$ but ranging up to $6 \%$. As before, higher ratios are found primarily in the vicinity of the L-Reactor discharge canal; however, three higher values were recorded within the old Steel Creek floodplain. It should be noted that the August 1995 ratios require an $8 \%$ decay correction for comparison with the 1996 ratios.
Three locations associated with the Steel Creek floodplain had high cobalt-60:cesium-137 ratios. Waypoints 38,43 , and 68 had ratios of $4.7 \%, 6.6 \%$, and $6.0 \%$, respectively. Waypoint 43 is fairly close to the L-Reactor discharge canal and thus its ratio corresponds with previous results. However, waypoints 38 and 68 are near the north and south ends of the lake, within sampling regions that generally have cobalt to cesium ratios measured at less than $2 \%$. No such aberrant ratios were observed 
Table 2. Results of cobalt-60:cesium-137 ratio analysis at 76 waypoints in L Lake. All results are decay corrected to April 24, 1996.

\begin{tabular}{|c|c|c|c|c|c|c|}
\hline Waypoint & $\begin{array}{l}\text { UTM' } \\
\text { North }\end{array}$ & $\begin{array}{l}\text { UTM } \\
\text { East }\end{array}$ & $\begin{array}{l}\text { Cobalt-60 } \\
\text { concentration } \\
\text { pCi/kg Error }\end{array}$ & $\begin{array}{l}\text { Cesium-137 } \\
\text { concentration } \\
\text { pCi/kg Error }\end{array}$ & \multicolumn{2}{|c|}{$\begin{array}{l}\text { Cobalt }-60: \\
\text { cesium-137 ratio } \\
\text { Percent } \quad \text { Error }\end{array}$} \\
\hline & & & & & & \\
\hline 6 & 3668 & 5.44 & $<8.1+1-$ MDA & $711+1 /$ & $<1.14$ & $+1-$ MDA \\
\hline 7 & 43 &  & $8.1+/-1.1$ & $1093+/-$ & 0.74 & $+1-0.10$ \\
\hline 8 & 2.87 & $\overline{81.41}$ & $<18.6+/-\mathrm{MDA}$ & $1053+/-22$ & $<1.77$ & $+/-M D A$ \\
\hline 9 & 3.04 & 0882.79 & $<23.3+/-\mathrm{MDA}$ & $1184+/-28$ & $<1.97$ & $+1-\overline{M D A}$ \\
\hline 10 & .55 & 2.17 & $18.3+/-4.8$ & $2071+/-$ & 0.88 & $+/-\quad-0.05$ \\
\hline 11 & 36694 & 40844.97 & $<3.4+1-\mathrm{MDA}$ & $138+1-$ & $<2.46$ & $+/-\quad M D A$ \\
\hline 14 & 3670200.41 & 441080.84 & $6.5+/-1.8$ & $1245+/-$ & 0.52 & $+/-\quad 0.15$ \\
\hline 15 & 3670251.66 & 441222.88 & $<8.2+1-\mathrm{MDA}$ & $442+1-$ & $<1.86$ & $+1-\mathrm{MDA}$ \\
\hline 16 & 3670168.22 & 441270.86 & $12.0+1-4.4$ & $1509+1-24$ & 0.80 & $+1-0.29$ \\
\hline 17 & 3670186.50 & 5.15 & $16.1+/-4.0$ & $1402+/-$ & 1.15 & $+/-\quad 0.29$ \\
\hline 19 & 6.74 & 4.41 & $<16.5+/-$ MDA & $990+1-20$ & $<1.67$ & $+/-\mathrm{MDA}$ \\
\hline 21 & 3669 & .23 & $75.1+/-15.5$ & $3788+1-\quad 88$ & 1.98 & $+/-0.41$ \\
\hline 22 & 7.92 & 2.69 & $<8.0+/-\quad 0.0$ & $435+1-$ & $<1.84$ & $+/-\quad M D A$ \\
\hline 23 & 70074.72 & 9.08 & $16.0+/-2.7$ & $1361+/-13$ & 1.18 & $+/-0.20$ \\
\hline 24 & 70013.03 & 66.20 & $<6.4+1-\mathrm{MDA}$ & $590+1-$ & $<1.08$ & $+1-\quad M D A$ \\
\hline 25 & 69920.93 & 79.63 & $<2.6+/-\mathrm{MDA}$ & $511+/-$ & $<0.51$ & $+1-\quad M D A$ \\
\hline 26 & 73650.89 & 2327.39 & $225.5+/-6.3$ & $13031+/-54$ & 1.73 & $+/-0.05$ \\
\hline 27 & 3673711.94 & 442315.63 & $11.6+1-1.8$ & $877+1-$ & 1.32 & $+/-0.20$ \\
\hline 28 & 3673802.25 & 442229.48 & $29.8+/-3.0$ & $2662+/-24$ & 1.12 & $+/-0.11$ \\
\hline 30 & 73827.30 & 442118.71 & $38.9+/-4.0$ & $3368+/-25$ & 1.15 & $+/-\quad 0.12$ \\
\hline 31 & 73852.13 & 442043.36 & $<4.5+/-\mathrm{MDA}$ & $516+/-27$ & $<0.87$ & $+/-\mathrm{MDA}$ \\
\hline 32 & 73924.63 & 71970.16 & $4.8+/-1.9$ & $310+1-$ & 1.55 & $+/-0.61$ \\
\hline 33 & 73866.82 & 71996.84 & $599.5+/-9.3$ & $11573+/-46$ & 5.18 & $+/-0.08$ \\
\hline 34 & 73736.96 & 442022.17 & $131.1+/-3.9$ & $4517+/-29$ & 2.90 & $+/-0.09$ \\
\hline 35 & 73641.68 & 442010.42 & $190.7+/-10.8$ & $11557+/-49$ & 1.65 & $+/-0.09$ \\
\hline 36 & 3673667.49 & 441959.30 & $62.0+/-2.3$ & $3919+/-34$ & 1.58 & $+/-0.06$ \\
\hline 37 & 3673756.42 & 441919.75 & $58.1+/-4.3$ & $2799+1-20$ & 2.08 & $+/-\quad 0.15$ \\
\hline 38 & 3673842.34 & 441827.06 & $120.2+/-7.9$ & $2573+/-39$ & 4.67 & $+/-\quad 0.32$ \\
\hline 39 & 3673842.60 & 441782.32 & $161.0+/-6.7$ & $4149+/-31$ & 3.88 & $+1-0.16$ \\
\hline 40 & 3673712.72 & 441810.44 & $125.8+/-7.5$ & $6430+1-38$ & 1.96 & $+/-\quad 0.12$ \\
\hline
\end{tabular}

a Any comparison to the 1995 data will require a decay correction to the August 1995 sample collection date.

b Universal Transverse Mercator.

c All less than $(<)$ values correspond to the Minimum Detectable Activities (MDA). 
Table 2 (continued). Results of cobalt-60:cesium-137 ratio analysis at 76 waypoints in L Lake. All results are decay corrected to April $24,1996 .^{a}$

\begin{tabular}{|c|c|c|c|c|c|c|c|}
\hline Waypoint & $\begin{array}{l}\text { UTM } \\
\text { North }\end{array}$ & $\begin{array}{l}\text { UTM } \\
\text { East }\end{array}$ & $\begin{array}{l}\text { Cobalt }-60 \\
\text { concentration }\end{array}$ & $\begin{array}{l}\text { Cesium-137 } \\
\text { concentration }\end{array}$ & \multicolumn{3}{|c|}{$\begin{array}{l}\text { Cobalt-60: } \\
\text { cesium-137 ratio }\end{array}$} \\
\hline & & & & & & & \\
\hline 41 & 3673640.55 & 441828.65 & $19.3+/-\quad 1.8$ & $1419+/$ & 1.36 & $+1-$ & 0.13 \\
\hline 42 & 3673479.37 & 441899.46 & $56.5+/-\quad 3.4$ & $3659+/-$ & 1.54 & $+/-$ & 0.09 \\
\hline$\overline{43}$ & 03.20 & 2.81 & $880.0+/-\quad 9.9$ & $13387+/-$ & 6.57 & $+1-$ & 0.08 \\
\hline 44 & 3674047.39 & 42771.53 & $19.5+/-2.1$ & $1266+/-$ & 1.54 & $+1-$ & 0.17 \\
\hline 45 & .51 & 2.72 & $50.0+/-\quad 7.0$ & $5765+/-$ & 0.87 & $+1-$ & 0.12 \\
\hline 46 & 3674042.67 & 442632.62 & $404.9+/-\quad 8.3$ & $9163+/-$ & 4.42 & $+/-$ & 0.09 \\
\hline 47 & 3675 & 00 & $57.6+1-4.2$ & $4589+1-$ & 1.26 & $+1-$ & 0.09 \\
\hline 48 & 3673882.52 & 442528.22 & $25.3+/-4.7$ & $1446+/-$ & 1.75 & $+1-$ & 0.32 \\
\hline 49 & 64.50 & .30 & $36.0+/-8.4$ & $3357+1-$ & 1.07 & $+\%$ & 0.25 \\
\hline 50 & 58.47 & 84 & $81.5+/-\quad 3.4$ & $4552+/-$ & 1.79 & $+1-$ & .08 \\
\hline 52 & 34.05 & 96 & $114.8+1-\quad 8.3$ & $11815+/-$ & 0.97 & $+1-$ & 0.07 \\
\hline 53 & 3672978.42 & 5.74 & $61.8+1-4.0$ & $3209+1-$ & 1.93 & $+1-$ & 0.13 \\
\hline 54 & 3672714.13 & 3.64 & $302.7+1-9.3$ & $14412+/-$ & 2.10 & $+1-$ & 0.07 \\
\hline 55 & 5.99 & 46 & $7.3+1-0.3$ & $1139+/-$ & 0.64 & $+1-$ & .02 \\
\hline 56 & 63.69 & 0.64 & $87.6+1-2.3$ & $5473+/-$ & 1.60 & $+1-$ & 0.04 \\
\hline 57 & 90.24 & 30 & $237.5+/-$ & $10906+/-$ & 2.18 & $+1-$ & .05 \\
\hline 58 & 3671443.47 & 7.13 & $<2.4+/-\mathrm{MDA}$ & $301+1-$ & $<0.80$ & $+1-$ & MDA \\
\hline 59 & 3671326.48 & 9.71 & $18.8+/-2.9$ & $1788+/-$ & 1.05 & $+1-$ & 0.16 \\
\hline 60 & 3671001.36 & 2.55 & $730.1+/-10.9$ & $47137+/-154$ & 1.55 & $+1-$ & 0.02 \\
\hline 62 & 3670526.60 & 74.42 & $183.5+/-10.9$ & $14448+/-$ & 1.27 & $+1-$ & 0.08 \\
\hline 63 & 45.04 & 3.18 & $6.1+/$ & $678+1-$ & 0.90 & $+1-$ & 0.22 \\
\hline 64 & 3670233.20 & 09.23 & $116.5+1-$ & $5669+/-$ & 2.06 & $+1-$ & 0.17 \\
\hline 65 & 3670074.59 & 0.39 & $=129.7+/-\mathrm{MDA}$ & $7095+/-148$ & $<1.83$ & $+1-$ & MDA \\
\hline 67 & 3670123.98 & 440702.74 & $37.8+/-4.5$ & $2956+1-$ & 1.28 & $+1-$ & 0.15 \\
\hline 68 & 3669794.31 & 7.88 & $887.5+/-14.2$ & $14699+/-$ & 6.04 & $+1-$ & 0.10 \\
\hline 69 & 3669427.80 & 5.69 & $19.4+/-\quad 1.2$ & $1338+1-$ & 1.45 & $+1-$ & 0.09 \\
\hline 70 & 3669219.32 & 83.75 & $10.0+1-2.0$ & $1000+/-$ & 1.00 & $+1-$ & 0.21 \\
\hline 71 & 3669178.14 & 0.55 & $65.2+/-5.8$ & $3793+1-$ & 1.72 & $+1-$ & 0.15 \\
\hline 72 & 3669172.08 & 95.37 & $23.3+/-7.3$ & $1581+/-$ & 1.47 & $+1-$ & 0.47 \\
\hline 73 & 3669249.67 & 97.70 & $90.1+/-9.1$ & $5315+/-$ & 1.70 & $+1-$ & 0.17 \\
\hline 74 & 3669238.63 & 440990.18 & $92.2+/-10.2$ & $5044+1-$ & 1.83 & $+1-$ & 0.20 \\
\hline 75 & 3669240.86 & 440988.33 & $97.4+/-\quad 4.7$ & $5277+1-$ & 1.85 & $+1-$ & 0.09 \\
\hline 76 & 3669330.25 & 441056.94 & $63.0+/-11.2$ & $3654+1-$ & 1.72 & +1 & 0.31 \\
\hline
\end{tabular}

a Any comparison to the 1995 data will require a decay correction to the August 1995 sample collection date.

b Universal Transverse Mercator.

c All less than $(<)$ values correspond to the Minimum Detectable Activities (MDA). 
in the 1995 survey (Appendix B). Since the present study had more than three times as many sediment samples as the 1995 survey, it may be that a larger number of samples provides a better opportunity to capture such anomalies. The 1985 overflight measurements were apparently not precise enough to locate such relatively infrequent variations in concentration.

Excluding the high ratios found at the L-Reactor discharge canal and the three high ratios in the Steel Creek floodplain, the remaining 57 samples averaged a cobalt- 60 :cesium- 137 ratio of $1.47 \% \pm$ $0.06 \%$, which, when decay-corrected to 1995 concentrations, yields a ratio of $1.59 \% \pm 0.07 \%$; this correlates with the $1.47 \% \pm 0.08 \%$, measured for August 1995 (Dunn et al. 1995). Uncertainty in the 1996 value can be attributed to including the larger minimal detectable activity values in the calculation of the average (causing the value to be higher), while at the same time omitting the high,outlier values from the Steel Creek floodplain in the calculation of the average (causing the value to be low).

The underwater HPGe detector did not count long enough to detect the lower cobalt-60 activities and produce a count-rate contour map; however, a map of cobalt 60 concentrations was produced from the 1985 overflight data (Figure 6; from Feimster 1992). Overall, the cobalt- 60 and cesium- 137 contour maps are similar. This is consistent with the relative uniformity of the majority of cobalt-60:cesium-137 ratios of 1-2\% shown in Figure 5. However, an exception to this is noted in the vicinity of the L-Reactor discharge channel, where the observed cobalt-60:cesium-137 ratios range up to $6 \%$.

The 1995 and 1996 measurements of cobalt- 60 and cesium-137 ratios appear to be consistent with the 1985 EG\&G overflight results. The 1985 overflight results show relatively more cobalt- 60 than cesium-137 near the L-Reactor discharge canal. This is consistent with the observed cobalt-60/cesium-137 ratio (based on 1995/1996 data) ranging to $6 \%$ near the discharge canal.

Most of the activation products released into SRS surface waters were transported directly to the Savannah River, although some cobalt-60 was deposited in stream beds, floodplains, and reservoirs (Gladden et al. 1985). L-Reactor basin purges were discharged to Steel Creek, L Lake, and a seepage basin designated 904-64G. The estimated aqueous release of cobalt-60 from $L$ Reactor to Steel Creek during the reactor's operational lifetime was 15 curies. Cumulative releases to seepage basins totaled 3.8 curies of cobalt-60 (Carlton et al. 1996).

Dissolved cobalt is relatively mobile, but migration can be attenuated by factors such as $\mathrm{pH}$ and coprecipitation with iron, manganese, or organic phases within the contaminant plume. It is reasonable to assume that cobalt- 60 may have preferentially accumulated near the $L$ discharge canal.

\section{Summary}

This study was conducted on L Lake on the SRS. L Lake was created in 1985 to dissipate heated effluent from L Reactor. Water from the Savannah River continues to be pumped to L Lake at a rate of 1.5 cubic meters per second in order to maintain the current water level. Pumping river water may no longer be needed to support current missions at SRS. Hydrologic models predict that $\mathrm{L}$ Lake would eventually drain if the river water pumping system is decommissioned.

In order to assess and understand the environmental impacts of lowering the L-Lake water level and exposing surface sediments, a four phase L-Lake site characterization plan was developed and implemented. Phase 3 involved the in situ measurement of gamma-emitting radionuclides in the submerged surface sediments of L Lake. Surface sediment samples also were collected for higher sensitivity HPGe gamma spectrometry in the Savannah River Technology Center. 


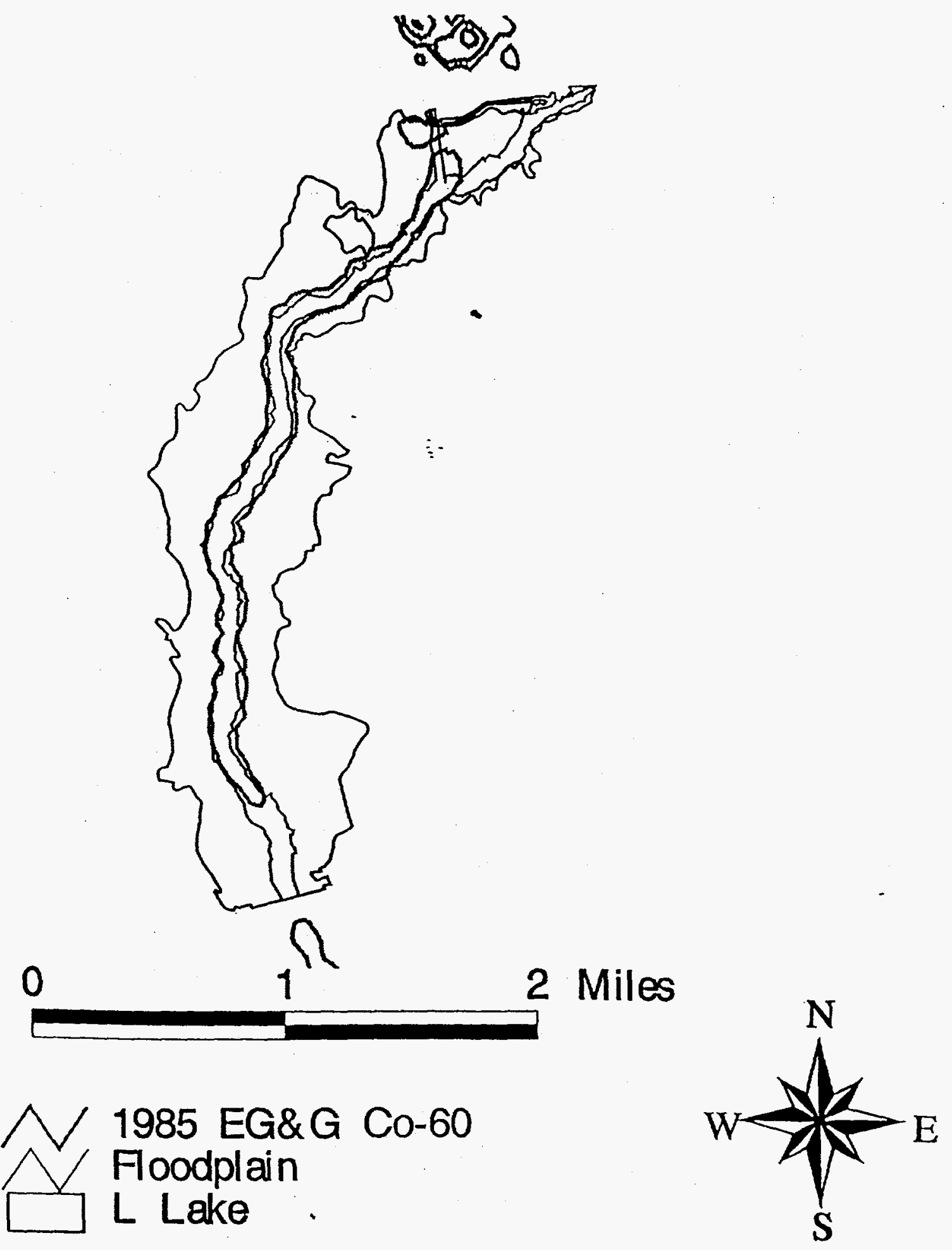

Figure 6. Cobalt-60 isodose contours from 1985 EG\&G overflight sodium iodide mapping (from Feimster 1992). 
The gamma spectral analysis results for all collected sediments and for all L-Lake measurements with the underwater HPGe detector are included in this report. The L-Lake cesium-137 activity is primarily located in the submerged Steel Creek floodplain. The collected sediment data show most cobalt-60:cesium137 ratios near $1-2 \%$ but ranging up to $6 \%$. The higher ratios are found primarily in the vicinity of the $\mathrm{L}-$ Reactor discharge canal, but some higher cobalt60:cesium-137 ratios also are evident further downstream in the submerged Steel Creek floodplain. These data are made available for a baseline risk assessment to evaluate potential human health risks of exposure to L-Lake sediments. 


\section{References}

Brisbin, I. L., Jr., R. J. Beyers, R. W. Dapson, R. A. Geiger, J. B. Gentry, J. W. Gibbons, M. H. Smith, and S. K. Woods. 1974. Patterns of Radiocesium in the Sediments of a Stream Channel Contaminated by Production Reactor Effluent. Health Physics. 27:19-27.

Carlton, W. H., L. R. Bauer, A. G. Evans, L. A. Geary, C. E. Murphy, Jr., J. E. Pinder, and R. N. Strom. 1992. Cesium in the Savannah River Site Environment. WSRC-RP-92-250. Westinghouse Savannah River Company, Savannah River Site, Aiken, SC.

DOE (U.S. Department of Energy). 1984. Final Environmental Impact Statement L-Reactor Operation Savannah River Plant. Vols 1-3. DOE/EIS0108. Savannah River Operations Office, Savannah River Plant, Aiken, SC.

DOE (U.S. Department of Energy). 1995. Environmental Assessment for the Natural Fluctuation of Water Level in Par Pond and Reduced Water Flow in Steel Creek Below L Lake at The Savannah River Site. DOE/EA-1070. Savannah River Operations Office, Aiken, SC.

D. L. Dunn, W. G. Winn, and P. J. Bresnahan. 1995. Scoping Measurements of Radionuclides in L Lake with an Underwater HPGe Detector (U). WSRCTR-95-0397. Westinghouse Savannah River Company, Savannah River Technology Center, Aiken, $\mathrm{SC}$.

Feimster, E. L. 1992. An Aerial Radiological Survey of the L Lake and Steel Creek, Savannah River Plant, Date of Survey: July 1986. EGG-106171146. EG\&G Energy Measurements, Inc., Las Vegas, NV.

Gladden, J. B., K. L. Brown, M. H. Smith, and A. Towns. 1985. Distribution of Gamma Exposure Rates in a Reactor Effluent Stream Flood Plain. Health Physics. 48:49-59.
Gladden, J. B., W. L. Specht, J. A. Bowers, N. V. Halverson, H. E. Mackey, and E. W. Wilde. 1989. Compliance of the Savannah River Plant L-Reactor Cooling System with Environmental Regulations $(U)$-Demonstration in Accordance with Section 316(a) of the Clean Water Act, November 1985-December 1987. DP-1766 Rev. 1. E. I. du Pont de Nemours \& Co., Savannah River Laboratory, Aiken, SC.

Tinney, L. R., C. E. Ezra, and H. E. Mackey, Jr. 1986. Stream Corridor and Delta Wetlands Change Assessments, Savannah River Plant, Aiken, SC. DOE (ONS-SRL)-8604. EG\&G/EM Letter Report, EG\&G Inc., Las Vegas, NV.

Winn, W. G., 1992. Evaluation of ${ }^{137} \mathrm{Cs}$ in Pond Sediment with an Underwater HPGe Detector, Trans. Amer. Nuc. Soc. 66:32-34.

Winn, W. G., 1993. Measurements of Radionuclides in Par Pond Sediments with an Underwater HPGe Detector. WSRC-TR-93-Q209. Westinghouse Savannah River Company, Aiken, SC.

Winn, W. G., 1995a. Environmental Measurements at the Savannah River Site with Underwater Gamma Detectors. Radioanalytical and Nuclear Chemistry. 194(2):345-350.

Winn, W. G., 1995b. Measurements of Radionuclides in Pond C with an Underwater HPGe Detector. WSRC-TR-95-0272. Westinghouse Savannah River Company, Aiken, SC.

Winn, W. G., W. W. Bowman, and A. L. Boni. 1987. Ultraclean Underground Counting Facility for Low-Level Environmental Samples. DP-1747. E. I. du Pont de Nemours \& Co., Aiken, SC.

Zeigler, C. C., I. B. Lawrimore, and W. E. O'Rear. 1985. Environmental Monitoring at the Savannah River Plant, Annual Report-1984. DPSPU 85-302. Health Protection Department, Savannah River Plant, E. I. du Pont de Nemours \& Co., Aiken, SC. 


\section{Appendix A \\ 1995 Underwater HPGe Data from L-Lake Sediments}

All entries are essentially self-explanatory; however, the following provides additional "Depth/ Loc" column information. The depth in feet may be translated to mean-sea-level (msl) depth by noting that the lake level is about $190 \mathrm{ft} \mathrm{msl}$; more exact levels may be deduced by checking the recorded lake level during the dates of the measure- ments. The location is given as "N," "S," " $E$," and "W" to denote relative compass directions relative to the deepest point of a transect; an " $M$ " denotes measurements near the lake middle. Notations " $a$ " and " $b$ " identify regions where additional dips (relative maximum depths) were observed. 


\section{Underwater HPGe Data - L Lake Surface Sediments (1995)}

\begin{tabular}{|c|c|c|c|c|c|c|c|}
\hline \multirow[t]{2}{*}{ Transect } & \multirow[t]{2}{*}{ Date } & \multirow[t]{2}{*}{ UTM north } & \multirow[t]{2}{*}{ UTM east } & \multirow{2}{*}{$\begin{array}{l}\text { Depth/ } \\
\text { Location }\end{array}$} & \multicolumn{2}{|c|}{ Cesium-137 } & rate \\
\hline & & & & & \multicolumn{3}{|c|}{ cpm } \\
\hline & & $\because \cdots$ & & $\beta \cdots$ & & m & 6 \\
\hline 1 & $8 / 1 / 95$ & 3673842.88 & 441775.79 & $3.5 \mathrm{ft} / \mathrm{W}$ & 1 & $+1-$ & 4 \\
\hline$\overline{1}$ & $8 / 1 / 95$ & 3673863.54 & 441823.04 & $8 \mathrm{ft} / \mathrm{W}$ & 88 & $+1-$ & 11 \\
\hline 1 & $8 / 1 / 95$ & 3673779.89 & 441855.94 & $12 \mathrm{ft} / \mathrm{W}$ & 11 & $+1-$ & 5 \\
\hline 1 & $8 / 1 / 95$ & 3673727.43 & 441925.01 & $16 \mathrm{ft} / \mathrm{W}$ & 6 & $+1-$ & 4 \\
\hline 1 & $8 / 1 / 95$ & 3673552.06 & 441948.83 & $20 \mathrm{ft} / \mathrm{W}$ & 257 & $+1-$ & 30 \\
\hline 1 & $8 / 1 / 95$ & 3673514.72 & 442012.56 & $24 \mathrm{ft} / \mathrm{W}$ & 122 & $+1-$ & 20 \\
\hline 1 & $8 / 1 / 95$ & 3673533.15 & 442021.21 & $25 \mathrm{ft} / \mathrm{W}$ & 117 & $+1-$ & 24 \\
\hline 1 & $8 / 1 / 95$ & 3673510.77 & 442055.00 & $20 \mathrm{ft} / \mathrm{E}$ & 8 & $+1-$ & 4 \\
\hline 1 & $8 / 1 / 95$ & 3673514.46 & 442108.36 & $12 \mathrm{ft} / \mathrm{E}$ & -2 & $+1-$ & 4 \\
\hline 2 & $8 / 1 / 95$ & 3673123.38 & 441075.23 & $3 \mathrm{ft} / \mathrm{W}$ & -1 & $+1-$ & 3 \\
\hline 2 & $8 / 1 / 95$ & 3673048.37 & 441103.26 & $11 \mathrm{ft} / \mathrm{W}$ & $\overline{0}$ & $+1-$ & 3 \\
\hline 2 & $8 / 1 / 95$ & 3673015.01 & 441120.92 & $20 \mathrm{ft} / \mathrm{W}$ & 4 & $+1-$ & 3 \\
\hline 2 & $8 / 1 / 95$ & 3673096.62 & 441120.12 & $24 \mathrm{ft} / \mathrm{W}$ & 1 & $+1-$ & 3 \\
\hline 2 & $8 / 1 / 95$ & 3673021.62 & 441402.42 & $12 \mathrm{ft} / \mathrm{Wa}$ & 7 & $+1-$ & 4 \\
\hline 2 & $8 / 1 / 95$ & 3673064.60 & 441374.46 & $20 \mathrm{ft} / \mathrm{Wa}$ & 13 & $+1-$ & 5 \\
\hline 2 & $8 / 1 / 95$ & 3673013.61 & 441351.63 & $30 \mathrm{ft} / \mathrm{Wa}$ & 15 & $+1-$ & 5 \\
\hline 2 & $8 / 1 / 95$ & 3672962.16 & 441406.73 & $36 \mathrm{ft} / \mathrm{Wa}$ & 2 & $+1-$ & 3 \\
\hline 2 & $8 / 1 / 95$ & 3673034.88 & 441502.45 & $34 \mathrm{ft} / \mathrm{Wb}$ & 39 & $+1-$ & 8 \\
\hline 2 & $8 / 1 / 95$ & 3672989.73 & 441482.76 & $30 \mathrm{ft} / \mathrm{Wb}$ & 381 & $+1-$ & 35 \\
\hline 2 & $8 / 1 / 95$ & 3672960.73 & 441531.70 & $26 \mathrm{ft} / \mathrm{Eb}$ & 48 & $+1-$ & 7 \\
\hline 2 & $8 / 1 / 95$ & 3672970.27 & 441569.67 & $22 f t / E b$ & 8 & $+1-$ & 3 \\
\hline 2 & $8 / 1 / 95$ & 3672935.46 & 441604.48 & $16 \mathrm{ft} / \mathrm{Eb}$ & 5 & $+1-$ & 3 \\
\hline 2 & $8 / 1 / 95$ & 3672941.79 & 441648.78 & $8 \mathrm{ft} / \mathrm{Eb}$ & 8 & $+1-$ & 3 \\
\hline 3 & $8 / 1 / 95$ & 3672615.03 & 441109.96 & $4 \mathrm{ft} / \mathrm{E}$ & -2 & $+1-$ & 3 \\
\hline 3 & $8 / 1 / 95$ & 3672579.35 & 441051.23 & $12 \mathrm{ft} / \mathrm{E}$ & 2 & $+1-$ & 1 \\
\hline 3 & $8 / 1 / 95$ & 3672555.83 & 441018.72 & $24 \mathrm{ft} / \mathrm{E}$ & 12 & $+1-$ & 4 \\
\hline 3 & $8 / 1 / 95$ & 3672546.12 & 440994.83 & $38 \mathrm{ft} / \mathrm{E}$ & 260 & $+1-$ & 16 \\
\hline 3 & $8 / 1 / 95$ & 3672472.18 & 440999.31 & $42 \mathrm{ft} / \mathrm{E}$ & 115 & $+1-$ & 12 \\
\hline 3 & $8 / 1 / 95$ & 3672393.74 & 440933.84 & $32 \mathrm{ft} / \mathrm{W}$ & 19 & $+1-$ & 5 \\
\hline 3 & $8 / 1 / 95$ & 3672550.88 & 440869.53 & $18 \mathrm{ft} / \mathrm{W}$ & 0 & $+1-$ & 2 \\
\hline 3 & $8 / 1 / 95$ & 3672631.51 & 440828.85 & $10 \mathrm{ft} / \mathrm{W}$ & 3 & $+1-$ & 3 \\
\hline 3 & $8 / 1 / 95$ & 3672545.00 & 440874.42 & $24 \mathrm{ft} / \mathrm{W}$ & 4 & $+1-$ & 2 \\
\hline 4 & $8 / 1 / 95$ & 3671934.33048 & 441331.15817 & $8 \mathrm{ft} / \mathrm{E}$ & 2 & $+1-$ & 1 \\
\hline 4 & $8 / 1 / 95$ & 3671862.48114 & 441142.19979 & $11 \mathrm{ft} / \mathrm{E}$ & 4 & $+1-$ & 3 \\
\hline 4 & $8 / 1 / 95$ & 3671884.66765 & 441088.98696 & $21 \mathrm{ft} / \mathrm{E}$ & 7 & $+1-$ & 3 \\
\hline 4 & $8 / 1 / 95$ & 3671973.74580 & 441025.30219 & $30 \mathrm{ft} / \mathrm{E}$ & 14 & $+1-$ & 4 \\
\hline 4 & $8 / 1 / 95$ & 3671958.56581 & 440989.21466 & $45 \mathrm{ft} / \mathrm{E}$ & 150 & $+1-$ & 12 \\
\hline 4 & $8 / 1 / 95$ & 3671836.22426 & 441002.45894 & $39 \mathrm{ft} / \mathrm{E}$ & 9 & $+1-$ & 3 \\
\hline 4 & $8 / 1 / 95$ & 3672057.33501 & 440851.00951 & $37 \mathrm{ft} / \mathrm{W}$ & 5 & +1 & 5 \\
\hline 4 & $8 / 1 / 95$ & 3672036.16944 & 440786.40004 & $30 \mathrm{ft} / \mathrm{W}$ & 4 & $+1-$ & 4 \\
\hline 4 & $8 / 1 / 95$ & 3672040.96456 & 440554.91848 & $29 \mathrm{ft} / \mathrm{W}$ & 2 & $+1-$ & 3 \\
\hline 4 & $8 / 1 / 95$ & 3672178.62697 & 440503.96580 & $16 \mathrm{ft} / \mathrm{W}$ & 4 & $+1-$ & 4 \\
\hline
\end{tabular}




\section{Underwater HPGe Data - L Lake Surface Sediments (1995) (continued)}

\begin{tabular}{|c|c|c|c|c|c|c|c|}
\hline \multirow[t]{2}{*}{ Transect } & \multirow[t]{2}{*}{ Date } & \multirow[t]{2}{*}{ UTM north } & \multirow[t]{2}{*}{ UTM east } & \multirow{2}{*}{$\begin{array}{l}\text { Depth/ } \\
\text { Location }\end{array}$} & \multicolumn{2}{|c|}{ Cesium-137 } & \multirow[t]{2}{*}{ rate } \\
\hline & & & & & & cpm & \\
\hline & & & & & & $\because$ & \\
\hline 5 & $8 / 2 / 95$ & 3670406.33355 & 440397.07040 & $14 \mathrm{ft} / \mathrm{W}$ & 2 & $+1-$ & 3 \\
\hline 5 & $8 / 2 / 95$ & 3670519.89952 & 440459.40598 & $24 \mathrm{ft} / \mathrm{W}$ & 12 & $+1-$ & 4 \\
\hline 5 & $8 / 2 / 95$ & 3670368.05560 & 440715.41278 & $32 \mathrm{ft} / \mathrm{W}$ & 0 & $+1-$ & 2 \\
\hline 5 & $8 / 2 / 95$ & 3670542.46213 & 440496.58064 & $40 \mathrm{ft} / \mathrm{W}$ & 1 & $+1-$ & 2 \\
\hline 5 & $8 / 2 / 95$ & 3670564.80687 & 440518.99081 & $45 \mathrm{ft} / \mathrm{W}$ & 7 & $+1-$ & 3 \\
\hline 5 & $8 / 2 / 95$ & 3670502.48435 & 440588.28246 & $51 \mathrm{ft} / \mathrm{W}$ & 3 & $+1-$ & 3 \\
\hline 5 & $8 / 2 / 95$ & 3670412.49317 & 440751.16612 & $55 \mathrm{ft} / \mathrm{W}$ & 6 & $+1-$ & 3 \\
\hline 5 & $8 / 2 / 95$ & 3670537.14348 & 440662.04950 & $56 \mathrm{ft} / \mathrm{W}$ & 3 & $+1-$ & 2 \\
\hline 5 & $8 / 2 / 95$ & 3670497.77084 & 440705.06342 & $59 \mathrm{ft} / \mathrm{W}$ & 29 & $+1-$ & 6 \\
\hline 5 & $8 / 2 / 95$ & 3670455.32201 & 440747.54101 & $56 \mathrm{ft} / \mathrm{E}$ & 49 & $+1-$ & 7 \\
\hline 5 & $8 / 2 / 95$ & 3670428.62194 & 440884.65062 & $36 \mathrm{ft} / \mathrm{E}$ & 3 & $+1-$ & 2 \\
\hline 5 & $8 / 2 / 95$ & 3670514.23194 & 440732.87650 & $54 \mathrm{ft} / \mathrm{M}$ & 5 & $+1-$ & 3 \\
\hline 5 & $8 / 2 / 95$ & 3670526.46220 & 440848.98300 & $36 \mathrm{ft} / \mathrm{Ea}$ & 11 & $+1-$ & 4 \\
\hline 5 & $8 / 2 / 95$ & 3670546.21797 & 440790.56870 & $44 \mathrm{ft} / \mathrm{Ea}$ & 5 & $+1-$ & 3 \\
\hline 5 & $8 / 2 / 95$ & 3670585.82781 & 440860.73866 & $12 \mathrm{ft} / \mathrm{E}$ & 7 & $+1-$ & 3 \\
\hline 6 & $8 / 2 / 95$ & 3669389.80954 & 440488.00177 & $6 \mathrm{ft} / \mathrm{W}$ & 1 & $+1-$ & 2 \\
\hline 6 & $8 / 7 / 95$ & 3669423.09775 & 440685.84449 & $15 \mathrm{ft} / \mathrm{W}$ & 3 & $+1-$ & 2 \\
\hline 6 & $8 / 7 / 95$ & 3669423.90202 & 440807.07534 & $36 \mathrm{ft} / \mathrm{W}$ & 4 & $+1-$ & 3 \\
\hline 6 & $8 / 7 / 95$ & 3669432.52253 & 440909.18523 & $45 \mathrm{ft} / \mathrm{W}$ & 2 & $+1-$ & 4 \\
\hline 6 & $8 / 7 / 95$ & 3669443.76550 & 441036.17754 & $68 \mathrm{ft} / \mathrm{W}$ & 142 & $+1-$ & 13 \\
\hline 6 & $8 / 7 / 95$ & 3669411.24503 & 440862.94913 & $63 \mathrm{ft} / \mathrm{W}$ & 15 & $+1-$ & 4 \\
\hline 6 & $8 / 7 / 95$ & 3669359.51351 & 441065.71649 & $54 \mathrm{ft} / \mathrm{E}$ & 2 & $+1-$ & 2 \\
\hline 6 & $8 / 7 / 95$ & 3669292.11342 & 441160.63395 & $23 \mathrm{ft} / \mathrm{E}$ & 0 & $+1-$ & 4 \\
\hline 6 & $8 / 7 / 95$ & 3669305.02714 & 441266.39708 & $15 \mathrm{ft} / \mathrm{E}$ & 3 & $+1-$ & 3 \\
\hline 6 & $8 / 7 / 95$ & 3669424.24271 & 441311.92612 & $4 \mathrm{ft} / \mathrm{E}$ & 2 & $+1-$ & 3 \\
\hline 7 & $8 / 2 / 95$ & 3669217.27 & 440856.07 & $48 \mathrm{ft} / \mathrm{M}$ & 5 & $+1-$ & 3 \\
\hline 7 & $8 / 2 / 95$ & 3669195.12 & 440902.82 & $45 \mathrm{ft} / \mathrm{W}$ & 6 & $+1-$ & 4 \\
\hline 7 & $8 / 2 / 95$ & 3669248.39 & 440902.89 & $68 \mathrm{ft} / \mathrm{W}$ & 5 & $+1-$ & 3 \\
\hline 7 & $8 / 2 / 95$ & 3669269.46 & 440984.61 & $68 \mathrm{ft} / \mathrm{E}$ & 6 & $+1-$ & 3 \\
\hline 7 & $8 / 2 / 95$ & 3669218.61 & 441040.77 & $70 \mathrm{ft} / \mathrm{E}$ & 3 & $+1-$ & 4 \\
\hline 8 & $8 / 7 / 95$ & 3670854.35 & 440762.65 & $42 \mathrm{ft} / \mathrm{E}$ & 12 & $+1-$ & 4 \\
\hline 8 & $8 / 7 / 95$ & 3670823.94 & 440697.46 & $48 \mathrm{ft} / \mathrm{E}$ & 7 & $+1-$ & 3 \\
\hline 8 & $8 / 7 / 95$ & 3670755.32 & 440689.79 & $57 \mathrm{ft} / \mathrm{E}$ & 9 & $+1-$ & 5 \\
\hline 8 & $8 / 7 / 95$ & 3670795.60 & 440547.07 & $47 \mathrm{ft} / \mathrm{E}$ & 8 & $+1-$ & 4 \\
\hline 8 & $8 / 7 / 95$ & 3670763.46 & 440769.09 & $51 \mathrm{ft} / \mathrm{E}$ & 7 & $+1-$ & 5 \\
\hline 9 & $8 / 7 / 95$ & 3671500.59 & 440486.36 & $32 \mathrm{ft} / \mathrm{W}$ & 12 & $+1-$ & 4 \\
\hline 9 & $8 / 7 / 95$ & 3671421.32 & 440608.89 & $47 \mathrm{ft} / \mathrm{W}$ & 8 & $+1-$ & 4 \\
\hline 9 & $8 / 7 / 95$ & 3671516.82 & 440654.53 & $49 \mathrm{ft} / \mathrm{W}$ & 194 & $+1-$ & 19 \\
\hline 9 & $8 / 7 / 95$ & 3671530.06 & 440805.60 & $27 \mathrm{ft} / \mathrm{E}$ & 10 & $+1-$ & 3 \\
\hline 10 & $8 / 7 / 95$ & 3673204.26 & 441967.47 & $5 \mathrm{ft} / \mathrm{S}$ & 0 & $+1-$ & 3 \\
\hline 10 & $8 / 7 / 95$ & 3673299.24 & 441997.29 & $4 \mathrm{ft} / \mathrm{N}$ & 3 & $+1-$ & 2 \\
\hline 10 & $8 / 7 / 95$ & & & $8 \mathrm{ft} / \mathrm{N}$ & 6 & $+1-$ & 3 \\
\hline 10 & $8 / 7 / 95$ & 3673241.60 & 442004.98 & $12 \mathrm{ft} / \mathrm{s}$ & 0 & $+1-$ & 3 \\
\hline 11 & $8 / 7 / 95$ & 3669807.37 & 441154.67 & $21 \mathrm{ft} / \mathrm{E}$ & 13 & $+1-$ & 4 \\
\hline 11 & $8 / 7 / 95$ & 3669788.13 & 441178.64 & $13 \mathrm{ft} / \mathrm{E}$ & 8 & $+1-$ & 4 \\
\hline 11 & $8 / 7 / 95$ & 3669815.76 & 441244.34 & $10 \mathrm{ft} / \mathrm{E}$ & 0 & $+1-$ & 2 \\
\hline 11 & $8 / 7 / 95$ & 3669718.05 & 441207.23 & $23 \mathrm{ft} / \mathrm{E}$ & 2 & $+1-$ & 4 \\
\hline 11 & $8 / 7 / 95$ & 3669558.69 & 441230.88 & $18 \mathrm{ft} / \mathrm{E}$ & 1 & $+1-$ & 1 \\
\hline
\end{tabular}


Underwater HPGe Data - L Lake Surface Sediments (1995) (continued)

\begin{tabular}{|c|c|c|c|c|c|c|c|}
\hline \multirow[t]{2}{*}{ Transect } & \multirow[t]{2}{*}{ Date } & \multirow[t]{2}{*}{ UTM north } & \multirow[t]{2}{*}{ UTM east } & \multirow{2}{*}{$\begin{array}{l}\text { Depth/ } \\
\text { Location }\end{array}$} & \multicolumn{2}{|c|}{ Cesium-137 } & rate \\
\hline & & & & & & $\mathrm{cpm}$ & \\
\hline \multicolumn{8}{|c|}{ - } \\
\hline $12-1$ & $8 / 9 / 95$ & 3670026.42 & 440883.51 & $25 \mathrm{ft} / \mathrm{M}$ & -1 & $+1-$ & $\frac{1}{2}$ \\
\hline $12-2$ & $8 / 9 / 95$ & 3670017.61 & 440863.77 & $30 \mathrm{ft} / \mathrm{M}$ & 13 & $+1-$ & 5 \\
\hline $12-3$ & $8 / 9 / 95$ & 3670113.85 & 440838.45 & $20 \mathrm{ft} / \mathrm{M}$ & -4 & $+1-$ & 3 \\
\hline $12-4$ & $8 / 9 / 95$ & 3670096.01 & 440988.05 & $17 \mathrm{ft} / \mathrm{M}$ & 1 & $+1-$ & 1 \\
\hline $12-5$ & $8 / 9 / 95$ & 3670035.33 & 440989.24 & $25 \mathrm{ft} / \mathrm{M}$ & 2 & $+1-$ & 2 \\
\hline $12-6$ & $8 / 9 / 95$ & 3669948.18 & 441040.25 & $48 \mathrm{ft} / \mathrm{M}$ & 1 & $+1-$ & $\overline{2}$ \\
\hline
\end{tabular}




\section{Appendix B \\ 1995 Gamma Spectrometry Data from L-Lake Sediments}

The 1995 sediment data analyzed in the Underground Counting Facility are summarized below. The table gives the following for each measurement:

All entries are essentially self-explanatory; however, it should be pointed out that the concentrations are for the undried sediments. (The dried sediments would have somewhat greater concentrations). In the interest of providing timely results for the 1995 scoping study, it was recognized that the data from the undried samples would be sufficient, as the prime interest was in examining the cobalt-60:cesium-137 ratio; however, even the absolute cobalt- 60 and cesium- 137 concentrations display the overall order-of-magnitude variations in the distribution, as the moisture content has a relatively minor effect on these trends.
An average of all the cobalt-60:cesium- 137 ratios yields $1.79 \% \pm 0.24 \%$, where the error is the standard deviation of the mean. (The corresponding sample standard deviation is $1.08 \%$ ). It should be noted that two ratios at $3.27 \%$ and $5.98 \%$ are significantly larger than the mean. Both values are in the vicinity of the L-Reactor discharge canal, where the earlier EG\&G overflight studies indicated relatively greater amounts of cobalt-60. Excluding these two values, the cobalt-60:cesium-137 average is $1.47 \%$ $\pm 0.08 \%$ (sample standard deviation of $0.32 \%$ ). This average value is considered more representative of the bulk of the lake, as the values making up this average span most locations of the lake and also cluster within a fairly narrow band about the average. 


\section{Gamma Spectrometry Data - L Lake Sedimentsa (1995)}

\begin{tabular}{|c|c|c|c|c|c|c|}
\hline $\begin{array}{l}\text { Sample } \\
\text { ID }\end{array}$ & $\begin{array}{l}\text { UTM } \\
\text { north }\end{array}$ & $\begin{array}{l}\text { UTM } \\
\text { east }\end{array}$ & Depth & $\begin{array}{l}\text { Cobalt-60 } \\
\mathrm{pCi} / \mathrm{kg}\end{array}$ & $\begin{array}{l}\text { Cesium-137 } \\
\mathrm{pCi} / \mathrm{kg}\end{array}$ & $\begin{array}{l}\text { Cobalt }-60 / \\
\text { Cesium }-137 \\
\text { percent }\end{array}$ \\
\hline \multicolumn{7}{|c|}{ का } \\
\hline $12-2$ & 3670017.61 & 440863.77 & $30 \mathrm{ft} / \mathrm{M}$ & $10.8+1-$ & $823+1-$ & $1.31+/-0.18$ \\
\hline $12-4$ & 3670096.01 & 440988.05 & $17 \mathrm{ft} / \mathrm{M}$ & $\overline{M D A}$ & $242+1-$ & 1.24 \\
\hline $12-6$ & 3669948.18 & 441040.25 & $48 \mathrm{ft} / \mathrm{M}$ & $9.5+1-$ & $593+1-$ & $1.60+/-0.23$ \\
\hline $13-1$ & 3669811.31 & 441061.96 & 28 feet & $3.3+1-$ & $179+/-$ & $1.84+/-0.43$ \\
\hline $13-2$ & 3669484.36 & 441299.59 & 56 feet & $12.6+1-$ & $836+/-10$ & $1.51+/-0.19$ \\
\hline $13-3$ & 3669613.33 & 441260.22 & 32 feet & $\overline{M D A}$ & $194+1-$ & 1.55 \\
\hline $13-4$ & 3669697.57 & 441335.32 & 14 feet & $5.3+1-$ & $338+1-$ & $1.57+/-0.28$ \\
\hline $13-5$ & 3669608.87 & 440572.74 & 14 feet & $\overline{M D A}$ & $268+1-$ & 1.08 \\
\hline $14-1$ & 3674125.94 & 442967.14 & 10 feet & $91.7+1-$ & $8190+1-20$ & $1.12+/-0.03$ \\
\hline $14-2$ & 3674026.77 & 442600.99 & 16 feet & $330.7+1-$ & $10100+1-200$ & $3.27+1-0.07$ \\
\hline $14-3$ & 3673397.66 & 442121.90 & 22 feet & $152.0+1-$ & $18100+/-100$ & $0.84+1-0.02$ \\
\hline $14-4$ & 3673711.47 & 441812.81 & 12 feet & $71.8+1-$ & $1200+/-10$ & $5.98+/-0.19$ \\
\hline $14-5^{b}$ & 3673014.20 & 441561.37 & 32 feet & $636.7+1-$ & $34400+1-100$ & $1.85+/-0.02$ \\
\hline $14-6$ & 3672614.21 & 441041.60 & 40 feet & $284.8+1-$ & $16200+/-200$ & $1.76+/-0.04$ \\
\hline $14-7$ & 3671917.03 & 440930.17 & 55 feet & $22.3+1-$ & $1550+/-10$ & $1.44+1-0.05$ \\
\hline $14-8$ & 3671796.54 & 441097.01 & 15 feet & $5.8+1-$ & $292+1-$ & $1.99+/-0.32$ \\
\hline $14-9$ & 3672103.96 & 440576.27 & 35 feet & $25.3+1-$ & $1370+/-\quad 10$ & $1.85+/-0.13$ \\
\hline $14-10$ & 3671090.01 & 440497.84 & 15 feet & $3.9+1-$ & $250+1-$ & $1.56+1-0.31$ \\
\hline 14-11 & 3670789.16 & 440796.96 & 54 feet & $23.2+1-$ & $1700+/-\quad 10$ & $1.36+1-0.09$ \\
\hline $14-12$ & 3669415.33 & 440747.71 & 24 feet & $7.5+/-$ & $742+1-$ & $1.01+/-0.17$ \\
\hline
\end{tabular}

a Analysis decay-corrected to sample collection date of August 9, 1995.

b Sample 14-5 indicated $28 \pm 5 \mathrm{pCi} / \mathrm{kg}$ for europium-154. 